\title{
Transient Taylor-Aris dispersion for time-dependent flows in straight channels
}

\author{
Vedel, Søren; Bruus, Henrik
}

Published in:

Journal of Fluid Mechanics

Link to article, DOI:

10.1017/jfm.2011.444

Publication date:

2012

Link back to DTU Orbit

Citation (APA):

Vedel, S., \& Bruus, H. (2012). Transient Taylor-Aris dispersion for time-dependent flows in straight channels. Journal of Fluid Mechanics, 691, 95-122. https://doi.org/10.1017/jfm.2011.444

\section{General rights}

Copyright and moral rights for the publications made accessible in the public portal are retained by the authors and/or other copyright owners and it is a condition of accessing publications that users recognise and abide by the legal requirements associated with these rights.

- Users may download and print one copy of any publication from the public portal for the purpose of private study or research.

- You may not further distribute the material or use it for any profit-making activity or commercial gain

- You may freely distribute the URL identifying the publication in the public portal

If you believe that this document breaches copyright please contact us providing details, and we will remove access to the work immediately and investigate your claim 


\title{
Transient Taylor-Aris dispersion for time-dependent flows in straight channels
}

\author{
Søren Vedel $\dagger$ and Henrik Bruus \\ Department of Micro- and Nanotechnology, Technical University of Denmark, \\ DTU Nanotech Building 345 B, DK-2800 Kongens Lyngby, Denmark
}

(Received 23 December 2010; revised 1 July 2011; accepted 6 October 2011; first published online 2 December 2011)

Taylor-Aris dispersion, the shear-induced enhancement of solute diffusion in the flow direction of the solvent, has been studied intensely in the past half century for the case of steady flow and single-frequency pulsating flows. Here, combining Aris's method of moments with Dirac's bra-ket formalism, we derive an expression for the effective solute diffusivity valid for transient Taylor-Aris dispersion in any given time-dependent, multi-frequency solvent flow through straight channels. Our theory shows that the solute dispersion may be greatly enhanced by the time-dependent parts of the flow, and it explicitly reveals how the dispersion coefficient depends on the external driving frequencies of the velocity field and the internal relaxation rates for mass and momentum diffusion. Although applicable to any type of fluid, we restrict the examples of our theory to Newtonian fluids, for which we both recover the known results for steady and single-frequency pulsating flows, and find new, richer structure of the dispersion as function of system parameters in multi-frequency systems. We show that the effective diffusivity is enhanced significantly by those parts of the time-dependent velocity field that have frequencies smaller than the fluid momentum diffusion rate and the solute diffusion rate.

Key words: general fluid mechanics, particle/fluid flows

\section{Introduction}

In his seminal work Taylor (1953) clarified the basic physical principles for the dispersion of the concentration profiles of solutes in a steady Poiseuille flow: the shear from the solvent flow acts to increase the dispersion, or effective diffusivity, of the solute in the direction of the flow. For a channel with a circular cross-section of radius $a$, he derived the now classical expression for the effective diffusivity $D_{\text {eff }}=\left(1+P e^{2} / 48\right) D$, where $P e^{\prime}=a U_{o} / D$ is the Péclet number for the system, $U_{o}$ being the average solvent flow speed and $D$ the solute molecular diffusivity. This work was extended to a wider range of Péclet numbers and geometries by Aris (1956) using the elegant method of statistical moments. Many other aspects of shear-enhanced solute dispersion in steady flows have since been studied in the literature, including buoyancy and channel curvature (Erdogan \& Chatwin 1967), interphase mass transfer (Sankarasubramanian \& Gill 1973), transient phenomena (Chatwin 1977), effects of walls in three-dimensional dispersion (Doshi, Daiya \& 
Gill 1978), a stochastic description (van den Broeck 1982), influence of channel aspect ratio (Chatwin \& Sullivan 1982), and generalized dispersion of mass, energy and momentum in unbounded systems (Brenner \& Edwards 1993; Goddard 1993). Taylor dispersion is now textbook material (Brenner \& Edwards 1993; Probstein 1994; Bird, Stewart \& Lightfoot 2006).

Even today shear-flow-enhanced dispersion is studied actively as illustrated by the recent analyses of $D_{\text {eff }}$ in transient anomalous diffusion of point discharges (Latini \& Bernoff 2001), in electro-osmotic flow with random zeta potentials (Gleeson 2002), in steady flows in a wide class of channel cross-sections (Ajdari, Bontoux \& Stone 2006; Bontoux et al. 2006; Dutta, Ramachandran \& Leighton 2006), in harmonically oscillating Couette-Poiseuille flows (Paul \& Mazumder 2008), in steady Poiseuille flows using a Brownian-motion approach (Camassa, Lin \& McLaughlin 2010) and in steady, non-Newtonian fluid flow (Vikhansky \& Wang 2011).

In many practical applications (Skafte-Pedersen et al. 2009; Vedel, Olesen \& Bruus 2010) flows are unsteady and therefore typically generate more shear than their steady counterparts. The chemical engineering community has long recognized that increased mass transfer can be achieved by pulsating the flow, see e.g. Taylor \& Leonard (1965) and Molloy \& Leighton (1998), but many physical interpretations and quantitative aspects of this additional shear remains to be investigated theoretically. This is surprising given that the first investigation of Taylor dispersion in time-dependent flows dates back to Aris (1960) and its generalization by Brenner \& Edwards (1993), and also given the large volume of studies dedicated to steady flows. Time-dependent phenomena that have been studied are non-transient, single-frequency pulsating flows (Harris \& Goren 1967; Chatwin 1975; Watson 1983; Thomas \& Narayanan 2001; Jansons 2006), the first examples of transient-flow analysis of single-frequency flows by Mazumder and coworkers (Mukherjee \& Mazumder 1988; Bandyopadhyay \& Mazumder 1999), and dispersion in a time-dependent flow in an unbounded system (Leighton 1989).

Here, we go beyond the previous results on dispersion in single-frequency flows and study any given time-dependent laminar flow in a straight channel of any constant cross-sectional shape, in particular relevant for microfluidic systems. Based on Aris's scheme we derive a general and compact expression for the transient, effective Taylor-Aris diffusivity $D_{\text {eff }}(t)$ with explicit dependence on the externally applied flow frequencies $\ell \omega_{o}$ and the internal relaxation rates $\lambda_{1}$ and $\alpha_{f l}$ for solute mass diffusion and solvent momentum diffusion, respectively. We show that $D_{\text {eff }}$ is enhanced significantly by those frequency-components of the time-dependent velocity field which have an oscillation frequency smaller than any relaxation rate of the system, and thus provide enough time for the solute to equilibrate. We further show how this enhancement is diminished each time a frequency is increased and becomes larger than a given relaxation rate. Moreover, for the start-up of a Poiseuille flow, which includes any flow frequency, we find that the transient time of $D_{\text {eff }}$ is determined by the slowest of the relaxation processes, i.e. the maximum of $1 / \lambda_{1}$ and $1 / \alpha_{f l}$. Finally, we demonstrate the practical usefulness of our theory as a design tool by characterizing the dispersion generated by a microfluidic, peristaltic pump.

\section{The physical model and Aris's method of moments}

In the following we establish our physical model together with our notation and present the well-known method of moments for calculating the dispersion coefficient $D_{\text {eff }}$. We consider a long, straight channel parallel to the $x$-axis, and assume that it is 
translational invariant along this axis with an arbitrary, but constant, cross-section $\Omega$. The coordinates in the transverse $y z$-plane are denoted $\boldsymbol{r}_{\perp}=(y, z)$, so that the full coordinates are written as $\boldsymbol{r}=\left(x, \boldsymbol{r}_{\perp}\right)$, and likewise for the gradient operator $\boldsymbol{\nabla}$ and the Laplace operator $\nabla^{2}$,

$$
\begin{aligned}
\boldsymbol{r} & =\left(x, \boldsymbol{r}_{\perp}\right) & & \text { with } \boldsymbol{r}_{\perp}=(y, z), \\
\nabla & =\boldsymbol{e}_{x} \partial_{x}+\nabla_{\perp} & & \text { with } \nabla_{\perp}=\boldsymbol{e}_{y} \partial_{y}+\boldsymbol{e}_{z} \partial_{z}, \\
\nabla^{2} & =\partial_{x}^{2}+\nabla_{\perp}^{2} & & \text { with } \nabla_{\perp}^{2}=\partial_{y}^{2}+\partial_{z}^{2} .
\end{aligned}
$$

The channel has length $\mathscr{L}$, cross-sectional area $\mathscr{A}=\int_{\Omega} \mathrm{d} \boldsymbol{r}_{\perp} 1$ and volume $\mathscr{V}=\mathscr{L} \mathscr{A}$. Our analysis involves expansion of the spatial dependence of functions into linear combinations of suitably chosen basis functions. For notational convenience we shall therefore rely on a Hilbert-space representation using the compact Dirac bra-ket notation, employed more often in quantum mechanics (Dirac 1981) than in fluid mechanics (Bruus 2008; Mortensen, Olesen \& Bruus 2006; Mortensen \& Bruus 2006). For any pair of functions $f\left(x, \boldsymbol{r}_{\perp}, t\right)$ and $g\left(x, \boldsymbol{r}_{\perp}, t\right)$ represented by the bra $\langle f|$ and the ket $|g\rangle$, the inner product $\langle f \mid g\rangle$, is defined by the integral, where the asterisk indicates complex conjugation,

$$
\langle f \mid g\rangle=\frac{1}{\mathscr{V}} \int_{-\mathscr{L} / 2}^{\mathscr{L} / 2} \mathrm{~d} x \int_{\Omega} \mathrm{d} \boldsymbol{r}_{\perp} f^{*}\left(x, \boldsymbol{r}_{\perp}, t\right) g\left(x, \boldsymbol{r}_{\perp}, t\right) .
$$

This definition also includes the case where the involved functions only depend on the transverse coordinates $\boldsymbol{r}_{\perp}$. For $f_{\perp}\left(\boldsymbol{r}_{\perp}, t\right)$ and $g_{\perp}\left(\boldsymbol{r}_{\perp}, t\right)$ we obtain

$$
\left\langle f_{\perp} \mid g_{\perp}\right\rangle=\frac{1}{\mathscr{A}} \int_{\Omega} \mathrm{d} \boldsymbol{r}_{\perp} f_{\perp}^{*}\left(\boldsymbol{r}_{\perp}, t\right) g_{\perp}\left(\boldsymbol{r}_{\perp}, t\right),
$$

as the $x$-integration trivially gives unity. Details and useful properties of the bra-ket notation are given in Appendix.

From now on we use dimensionless quantities defined in terms of the characteristic transverse length $L_{o}$, often the shortest distance $a$ from the centre line of the channel to the wall, the diffusion time $T_{o}$ from the molecular diffusivity $D$ of the solute, the advection velocity $U_{o}$ from a scale $U_{\text {char }}$ in the time-dependent flow, and the average solute concentration $C_{o}$ of the solute concentration field $c\left(x, \boldsymbol{r}_{\perp}, t\right)$,

$$
L_{o}=a, \quad T_{o}=\frac{L_{o}^{2}}{D}, \quad U_{o}=U_{\text {char }}, \quad C_{o}=\left\langle 1 \mid c\left(x, \boldsymbol{r}_{\perp}, 0\right)\right\rangle .
$$

The specific choice of $U_{\text {char }}$ in (2.4) is not unique: for a steady flow it could be taken as the mean velocity; for a single-frequency oscillating flow as the root mean square (r.m.s.) velocity. See Appendix for a list of symbols.

Denoting the base angular frequency of the solvent flow $\tilde{\omega}_{o}\left(\operatorname{rad~s}^{-1}\right)$, the system is characterized by three dimensionless numbers: the Péclet number Pé (the ratio of advection speed to mass diffusion speed), the Schmidt number $S c$ (the ratio of momentum diffusion speed to mass diffusion speed) and the Womersley number Wo (the square root of the ratio of oscillation speed at frequency $\tilde{\omega}_{o}$ to momentum diffusion speed for a Newtonian fluid of kinematic viscosity v),

$$
P e ́=\frac{L_{o} U_{o}}{D}, \quad S c=\frac{v}{D}, \quad W o=\sqrt{\frac{L_{o}^{2} \tilde{\omega}_{o}}{v}} .
$$


For non-Newtonian fluids with an internal molecular stress relaxation time $\tau_{m o l}$, the Deborah number $D e=\tau_{m o l} \omega_{o}$ would appear, but this is not treated in this work.

\subsection{The time-dependent velocity field}

We take the velocity field to be any axis-parallel channel flow $\boldsymbol{v}=u\left(\boldsymbol{r}_{\perp}, t\right) \boldsymbol{e}_{x}$, and represent $u\left(\boldsymbol{r}_{\perp}, t\right)$ by a standard Fourier series with components $u_{\ell}$ for each higher harmonic $\ell \omega_{o}(\ell$ being an integer) in the dimensionless base frequency $\omega_{o}=\tilde{\omega}_{o} T_{o}=W o^{2} S c$,

$$
u\left(\boldsymbol{r}_{\perp}, t\right)=\sum_{\ell=-\infty}^{\infty} u_{\ell}\left(\boldsymbol{r}_{\perp}\right) \mathrm{e}^{\mathrm{i} \ell \omega_{o} t},
$$

where complex notation is introduced for the time with $\mathrm{i}=\sqrt{-1}$. By demanding that $u_{-\ell}\left(\boldsymbol{r}_{\perp}\right)=u_{\ell}^{*}\left(\boldsymbol{r}_{\perp}\right)$, we ensure that the velocity field is real (Brenner \& Edwards 1993).

The fluid responds to changes in external conditions on a time scale which depends on its internal stress relaxation time, and whether fluid inertia dominates may be estimated by the product of the driving frequency $\tilde{\omega}_{o}$ and this internal time scale. For Newtonian fluids the stress relaxation time scale is given by the momentum diffusion time $1 / \alpha_{f l}$, related to the momentum diffusion rate $\alpha_{f l}$, which is derived from the momentum diffusion equation $\partial_{t} u=S c \nabla_{\perp}^{2} u$ and given by

$$
\alpha_{f l} \propto S c
$$

with a geometry-dependent proportionality factor. Note that since $\alpha_{f l} \propto S c$, the square of the Womersley number is proportional to the ratio of the dimensionless driving frequency $\omega_{o}$ and the momentum diffusion equilibration rate,

$$
W o^{2}=\frac{\omega_{o}}{S c} \propto \frac{\omega_{o}}{\alpha_{f l}} .
$$

\subsection{Dispersion and the advection-diffusion equation}

The transport of the diffusive solute concentration $c$ in the above-mentioned velocity field is given by the dimensionless advection-diffusion equation,

$$
\partial_{t} c\left(x, \boldsymbol{r}_{\perp}, t\right)+P e ́ u\left(\boldsymbol{r}_{\perp}, t\right) \partial_{x} c\left(x, \boldsymbol{r}_{\perp}, t\right)=\left(\partial_{x}^{2}+\nabla_{\perp}^{2}\right) c\left(x, \boldsymbol{r}_{\perp}, t\right) .
$$

The corresponding boundary conditions are

$$
\begin{aligned}
\boldsymbol{n} \cdot \nabla_{\perp} c & =0, \text { on all walls, } \\
c\left(x, \boldsymbol{r}_{\perp}, 0\right) & =\tilde{c}\left(x, \boldsymbol{r}_{\perp}\right), \\
x^{s} \partial_{x}^{q} c & \rightarrow 0, \quad \text { for }|x| \rightarrow \frac{\mathscr{L}}{2} \text { and } s, q=0,1,2, \ldots,
\end{aligned}
$$

where $\boldsymbol{n}$ is the surface normal and $\tilde{c}$ is a given initial concentration field, and condition (2.10c) states that all spatial gradients in $c$ as well as $c$ itself vanish far away along the axis of the channel.

Using Aris's method of moments the effective diffusivity $D_{\text {eff }}(t)$ is defined by the time derivative of the axial variance $\mu_{2}(t)=\left\langle(x-\bar{x})^{2} \mid c\right\rangle$ of the solute distribution, $\bar{x}(t)=M_{1}(t)$ being the centre of mass (A $\left.5 b\right)$, and may be computed as (Aris 1956, 1960; Chatwin 1975; Barton 1983; Mukherjee \& Mazumder 1988; Brenner 
\& Edwards 1993)

$$
D_{\text {eff }}(t)=\frac{1}{2} \frac{\mathrm{d} \mu_{2}(t)}{\mathrm{d} t}=\frac{1}{2} \frac{\mathrm{d} M_{2}}{\mathrm{~d} t}-M_{1} \frac{\mathrm{d} M_{1}}{\mathrm{~d} t},
$$

where the $p$ th full moment $M_{p}(t)$ of the solute concentration field $c\left(x, \boldsymbol{r}_{\perp}, t\right)$ and the associated axial moment $c_{p}\left(\boldsymbol{r}_{\perp}, t\right)$ are defined by

$$
\begin{aligned}
& M_{p}(t)=\left\langle x^{p} \mid c\right\rangle=\frac{1}{\mathscr{A}} \int_{\Omega} \mathrm{d} \boldsymbol{r}_{\perp} c_{p}\left(\boldsymbol{r}_{\perp}, t\right), \quad p=0,1,2, \ldots, \\
& c_{p}\left(\boldsymbol{r}_{\perp}, t\right)=\frac{1}{\mathscr{L}} \int_{-\mathscr{L} / 2}^{\mathscr{L} / 2} \mathrm{~d} x x^{p} c\left(x, \boldsymbol{r}_{\perp}, t\right), \quad p=0,1,2, \ldots
\end{aligned}
$$

The derivation of (2.11) is sketched in Appendix, where we also show that the moments $c_{p}\left(\boldsymbol{r}_{\perp}, t\right)$ and $M_{p}(t)$ evolve according to the recursive equations of motion,

$$
\begin{gathered}
\partial_{t} c_{p}\left(\boldsymbol{r}_{\perp}, t\right)-\nabla_{\perp}^{2} c_{p}\left(\boldsymbol{r}_{\perp}, t\right)=p(p-1) c_{p-2}\left(\boldsymbol{r}_{\perp}, t\right)+P^{\prime} u\left(\boldsymbol{r}_{\perp}, t\right) p c_{p-1}\left(\boldsymbol{r}_{\perp}, t\right), \\
\quad p=0,1,2, \ldots, \\
\frac{\mathrm{d} M_{p}(t)}{\mathrm{d} t}=p(p-1)\left\langle 1 \mid c_{p-2}\right\rangle+P e ́ p\left\langle u \mid c_{p-1}\right\rangle, \\
p=0,1,2, \ldots,
\end{gathered}
$$

with the boundary and initial conditions

$$
\begin{aligned}
\boldsymbol{n} \cdot \nabla_{\perp} c_{p} & =0 \quad \text { on all walls, } \\
c_{p}\left(\boldsymbol{r}_{\perp}, t\right) & <\infty \\
c_{p}\left(\boldsymbol{r}_{\perp}, 0\right) & =\tilde{c}_{p}\left(\boldsymbol{r}_{\perp}\right), \\
M_{p}(0) & =\left\langle x^{p} \mid \tilde{c}\right\rangle .
\end{aligned}
$$

The main goal of this paper is to solve (2.11) for general time-dependent flows in straight channels of arbitrary, constant cross-section, and to study how $D_{\text {eff }}$ depends on the physical frequencies and relaxation rates of the systems.

\section{Dispersion for multiple-frequency flow}

The solution procedure of the problem was introduced by Barton (1983) for steady flows and later extended to also include single-frequency harmonic pulsatile flows by Mukherjee \& Mazumder (1988). The expressions presented in this section generalize their results to any given time-dependent flow. We derive them using the more compact bra-ket formalism as follows: from (2.13a) with $p=0$ and 1 we determine the axial moment $c_{o}\left(\boldsymbol{r}_{\perp}, t\right)$ and subsequently $c_{1}\left(\boldsymbol{r}_{\perp}, t\right)$. With these at hand, the full moment $M_{1}$ as well as the time derivatives $\mathrm{d} M_{1} / \mathrm{d} t$ and $\mathrm{d} M_{2} / \mathrm{d} t$ can be obtained from (2.13b) with $p=1$ and 2 , which then are fed into (2.11) to determine $D_{\text {eff }}$.

\subsection{The zeroth axial moment and basis functions}

We begin by analysing the $p=0$ axial moment (2.13a) for $c_{o}\left(\boldsymbol{r}_{\perp}, t\right)$,

$$
\left(\partial_{t}-\nabla_{\perp}^{2}\right)\left|c_{o}\left(\boldsymbol{r}_{\perp}, t\right)\right\rangle=0 .
$$

This moment fulfills the Neumann boundary condition

$$
\boldsymbol{n} \cdot \nabla_{\perp} c_{o}=0 \quad \text { on all walls, }
$$


and has the initial condition

$$
c_{o}\left(\boldsymbol{r}_{\perp}, 0\right)=\tilde{c}_{0}\left(\boldsymbol{r}_{\perp}\right)=\int_{-\mathscr{L} / 2}^{\mathscr{L} / 2} \mathrm{~d} x \tilde{c}\left(x, \boldsymbol{r}_{\perp}\right) .
$$

Using separation of variables, the solution for $c_{o}\left(\boldsymbol{r}_{\perp}, t\right)$ can be written as the expansion

$$
\left|c_{o}\left(\boldsymbol{r}_{\perp}, t\right)\right\rangle=\sum_{n=0}^{\infty} a_{0 n} \mathrm{e}^{-\lambda_{n} t}\left|f_{n}\left(\boldsymbol{r}_{\perp}\right)\right\rangle,
$$

where the time-independent eigenfunctions $f_{n}\left(\boldsymbol{r}_{\perp}\right)$ with eigenvalues $\lambda_{n}$ are defined by

$$
\begin{aligned}
\left(\lambda_{n}+\nabla_{\perp}^{2}\right)\left|f_{n}\left(\boldsymbol{r}_{\perp}\right)\right\rangle & =0, \quad n=0,1,2, \ldots, \\
\boldsymbol{n} \cdot \nabla_{\perp}\left|f_{n}\left(\boldsymbol{r}_{\perp}\right)\right\rangle & =0 \quad \text { on all walls, }
\end{aligned}
$$

and form a complete orthonormal basis in the sense of (A 3). Note that the lowest $n=0$ eigenvalue is zero, $\lambda_{0}=0$, with eigenfunction unity, $f_{0}\left(\boldsymbol{r}_{\perp}\right)=1$, while for $n>0$ the eigenvalues are positive, $\lambda_{n}>0$. We remark that although the general advection-diffusion problem (2.9) is non-Hermitian, the reduced, transverse problem (3.5) is Hermitian, and we can take full advantage of the Dirac Hilbert-space formulation.

The expansion coefficients $a_{0 m}$ are found by multiplying (3.4) at $t=0$ by $\left\langle f_{m}\right|$,

$$
a_{0 m}=\left\langle f_{m} \mid \tilde{c}_{0}\right\rangle, \quad m=0,1,2, \ldots
$$

For infinite time, all terms in (3.4) except $n=0$ decay exponentially, and we obtain

$$
\left|c_{o}\left(\boldsymbol{r}_{\perp}, \infty\right)\right\rangle=a_{00}\left|f_{0}\left(\boldsymbol{r}_{\perp}\right)\right\rangle=\left\langle 1 \mid \tilde{c}_{0}\right\rangle|1\rangle=|1\rangle,
$$

representing the state where, by diffusion, the solute concentration has spread out uniformly in space.

\subsection{The first axial moment and basis functions}

The $p=1$ axial moment equation (2.13a) for $c_{1}\left(\boldsymbol{r}_{\perp}, t\right)$ is analysed in a similar manner. Using that $c_{o}\left(\boldsymbol{r}_{\perp}, t\right)$ is now a known function, we have

$$
\left(\partial_{t}-\nabla_{\perp}^{2}\right)\left|c_{1}\left(\boldsymbol{r}_{\perp}, t\right)\right\rangle=\operatorname{Pé} u\left(\boldsymbol{r}_{\perp}, t\right)\left|c_{o}\left(\boldsymbol{r}_{\perp}, t\right)\right\rangle=\operatorname{Pé} u\left(\boldsymbol{r}_{\perp}, t\right) \sum_{n=0}^{\infty} a_{0 n} \mathrm{e}^{-\lambda_{n} t}\left|f_{n}\left(\boldsymbol{r}_{\perp}\right)\right\rangle,
$$

where $c_{1}$ fulfills the Neumann boundary condition

$$
\boldsymbol{n} \cdot \nabla_{\perp} c_{1}=0 \quad \text { on all walls, }
$$

and the initial condition

$$
c_{1}\left(\boldsymbol{r}_{\perp}, 0\right)=\tilde{c}_{1}\left(\boldsymbol{r}_{\perp}\right)=\int_{-\mathscr{L} / 2}^{\mathscr{L} / 2} \mathrm{~d} x x \tilde{c}\left(x, \boldsymbol{r}_{\perp}\right) .
$$

As pointed out by Barton (1983), solving the inhomogeneous partial differential equation (3.8a) for $c_{1}$ requires some care regarding the null space of the differential operator $\left(\partial_{t}-\nabla_{\perp}^{2}\right)$. Using the time Fourier expansion equation (2.6) of the velocity field $u\left(\boldsymbol{r}_{\perp}, t\right)$, we see that, due to the time-independent $\ell=0$ component of $u$, the right-hand side of (3.8a) contains terms of the form Pé $a_{0 n} u_{0}\left(\boldsymbol{r}_{\perp}\right) \mathrm{e}^{-\lambda_{n} t}\left|f_{n}\right\rangle$, which have 

non-zero overlap with kets of the form $\mathrm{e}^{-\lambda_{n} t}\left|f_{n}\right\rangle$. Noting that

$$
\begin{aligned}
\left(\partial_{t}-\nabla_{\perp}^{2}\right)\left[\mathrm{e}^{-\lambda_{n} t}\left|f_{n}\right\rangle\right] & =0, \\
\left(\partial_{t}-\nabla_{\perp}^{2}\right)\left[t \mathrm{e}^{-\lambda_{n} t}\left|f_{n}\right\rangle\right] & =\mathrm{e}^{-\lambda_{n} t}\left|f_{n}\right\rangle,
\end{aligned}
$$

we thus expand $c_{1}\left(\boldsymbol{r}_{\perp}, t\right)$ in terms of both $\mathrm{e}^{-\lambda_{n} t}\left|f_{n}\right\rangle$ and $t \mathrm{e}^{-\lambda_{n} t}\left|f_{n}\right\rangle$, and seek solutions of $\left|c_{1}\left(\boldsymbol{r}_{\perp}, t\right)\right\rangle$ of the form

$$
\left|c_{1}\right\rangle=P e \sum_{n=0}^{\infty}\left[\left(\gamma_{1 n} a_{0 n} t+a_{1 n}\right)\left|f_{n}\right\rangle+a_{0 n}\left|\phi_{n}\right\rangle\right] \mathrm{e}^{-\lambda_{n} t}
$$

where the unknown coefficients, $\gamma_{1 n}$ and $a_{1 n}$, as well as the unknown time-dependent ket $\left|\phi_{n}\left(\boldsymbol{r}_{\perp}, t\right)\right\rangle$ are determined in the following. Inserting this trial expansion of $\left|c_{1}\left(\boldsymbol{r}_{\perp}, t\right)\right\rangle$ into the equation of motion (3.8a) leads to

$$
\left(\partial_{t}-\nabla_{\perp}^{2}-\lambda_{n}\right)\left|\phi_{n}\left(\boldsymbol{r}_{\perp}, t\right)\right\rangle=\left[u\left(\boldsymbol{r}_{\perp}, t\right)-\gamma_{1 n}\right]\left|f_{n}\left(\boldsymbol{r}_{\perp}\right)\right\rangle .
$$

The unknown ket $\left|\phi_{n}\left(\boldsymbol{r}_{\perp}, t\right)\right\rangle$ is now expanded in a Fourier series in time and a $\left|f_{m}\left(\boldsymbol{r}_{\perp}\right)\right\rangle$ series in space,

$$
\left|\phi_{n}\left(\boldsymbol{r}_{\perp}, t\right)\right\rangle=\sum_{\ell=-\infty}^{\infty} \sum_{m=0}^{\infty} \beta_{m n}^{\ell} \mathrm{e}^{\mathrm{i} \ell \omega_{o} t}\left|f_{m}\left(\boldsymbol{r}_{\perp}\right)\right\rangle
$$

which upon insertion into (3.11) followed by multiplication by $\left\langle f_{k}\right|$ results in the following matrix equation for the coefficients $\beta_{k n}^{\ell}$,

$$
\left(\lambda_{k}-\lambda_{n}+\mathrm{i} \ell \omega_{o}\right) \beta_{k n}^{\ell}=\left\langle f_{k}\left|u_{\ell}\right| f_{n}\right\rangle-\gamma_{1 n} \delta_{\ell, 0} \delta_{k, n} .
$$

Here, we see that the special case of the time-independent term $\ell=0$, together with the diagonal term $k=n$, only allows a solution if we choose

$$
\gamma_{1 n}=\left\langle f_{n}\left|u_{0}\left(\boldsymbol{r}_{\perp}\right)\right| f_{n}\right\rangle
$$

while the $\beta$ coefficients are given by

$$
\beta_{k n}^{\ell}=\left(1-\delta_{\ell, 0} \delta_{k, n}\right) \frac{\left\langle f_{k}\left|u_{\ell}\left(\boldsymbol{r}_{\perp}\right)\right| f_{n}\right\rangle}{\lambda_{k}-\lambda_{n}+\mathrm{i} \ell \omega_{o}} .
$$

Note that any value of $\beta_{n n}^{0}$ is allowed, so for convenience we set it to zero. Moreover, $\beta_{k n}^{-\ell}=\left(\beta_{k n}^{\ell}\right)^{*}$ ensures real values of the resulting fields.

Finally, the coefficients $a_{1 n}$ are found using the initial condition (3.8c) in (3.10) at $t=0$, multiplying by $\left\langle f_{k}\right|$, and finally exchanging the indices $n$ and $k$,

$$
a_{1 n}=\frac{1}{P e ́}\left\langle f_{n} \mid \tilde{c}_{1}\left(\boldsymbol{r}_{\perp}\right)\right\rangle-\sum_{k=0}^{\infty} a_{0 k} \sum_{l=-\infty}^{\infty} \beta_{n k}^{\ell} .
$$

Collecting all terms, we write the formal solution as

$$
\left|c_{1}\left(\boldsymbol{r}_{\perp}, t\right)\right\rangle=P e ́ \sum_{m=0}^{\infty} \sum_{n=0}^{\infty}\left[\left(a_{0 n} \gamma_{1 n} t+a_{1 n}\right) \delta_{n, m}+a_{0 n}\left(\sum_{\ell=-\infty}^{\infty} \beta_{m n}^{\ell} \mathrm{e}^{\mathrm{i} \ell \omega_{o} t}\right)\right] \mathrm{e}^{-\lambda_{n} t}\left|f_{m}\right\rangle
$$




\subsection{Expressions for the effective diffusivity}

The effective diffusivity $D_{\text {eff }}=(1 / 2)\left(\mathrm{d} M_{2} / \mathrm{d} t\right)-M_{1}\left(\mathrm{~d} M_{1} / \mathrm{d} t\right)$ can now be expressed in terms of a basis function expansion. The time derivative $\mathrm{d} M_{1} / \mathrm{d} t=P e ́\left(u\left|c_{o}\right\rangle\right.$ of the full moment $M_{1}$ in (2.13b) becomes

$$
\frac{\mathrm{d} M_{1}}{\mathrm{~d} t}=P e ́ \sum_{n=0}^{\infty} \sum_{\ell=-\infty}^{\infty} a_{0 n}\left\langle u_{\ell} \mid f_{n}\right\rangle \mathrm{e}^{-\left(\lambda_{n}+\mathrm{i} \ell \omega_{o}\right) t},
$$

where we have used (A 2b) for the phase factor. By proper choice of the coordinate system, the initial centroid $\bar{x}(0)$ of the distribution is zero. This, combined with (A $5 b)$, determines the initial value $M_{1}(0)=\bar{x}(0)=0$, and time integration of $(3.18 a)$ gives

$$
M_{1}=P e^{\prime} \sum_{n=0}^{\infty} \sum_{\ell=-\infty}^{\infty} a_{0 n}\left\langle u_{\ell} \mid f_{n}\right\rangle \frac{1-\mathrm{e}^{-\left(\lambda_{n}+\mathrm{i} \ell \omega_{o}\right) t}}{\lambda_{n}+\mathrm{i} \ell \omega_{o}} .
$$

Here, the term $(n, \ell)=(0,0)$ depends linearly on time because $\lim _{q \rightarrow 0}\left[\left(1-\mathrm{e}^{-q t}\right) / q\right]=t$. Similarly, for the time derivative $(1 / 2)\left(\mathrm{d} M_{2} / \mathrm{d} t\right)=\left\langle 1 \mid c_{o}\right\rangle+P e ́\left(u\left|c_{1}\right\rangle\right.$ of the full moment $M_{2},(2.13 b)$, we obtain

$$
\begin{aligned}
\frac{1}{2} \frac{\mathrm{d} M_{2}}{\mathrm{~d} t}= & 1+P e^{-2} \sum_{m=0}^{\infty} \sum_{n=0}^{\infty} \sum_{k=-\infty}^{\infty}\left\langle u_{k} \mid f_{m}\right\rangle \mathrm{e}^{-\left(\lambda_{n}+\mathrm{i} k \omega_{o}\right) t} \\
& \times\left[\left(a_{0 n} \gamma_{1 n} t+a_{1 n}\right) \delta_{n, m}+a_{0 n} \sum_{\ell=-\infty}^{\infty} \beta_{m n}^{\ell} \mathrm{e}^{i \ell \omega_{o} t}\right] .
\end{aligned}
$$

All expressions derived so far are explicitly real because of the pairwise summation of complex conjugate terms with index $\ell$ and $-\ell$. Furthermore, they apply to any initial solute distributions, such as the non-uniform distributions recently studied by Camassa et al. (2010), as well as any given velocity field, which can be represented by (2.6).

We now study the special case of an initial solute distribution $\tilde{c}$ being uniform in the cross-sectional plane. This introduces significant simplifications in $D_{\text {eff }}$, which otherwise depends on the channel cross-section, the flow profile $u$, and the initial solute distribution $\tilde{c}$ through the coefficients $u_{\ell}, a_{0 n}, a_{1 n}, \gamma_{1 n}$, and $\beta_{k n}^{\ell}$. Transverse uniformity leads to

$$
\left|\tilde{c}_{0}\right\rangle=|1\rangle \quad \text { and } \quad\left|\tilde{c}_{1}\right\rangle=0
$$

and $a_{0 n}, \beta_{j 0}^{\ell}$ and $a_{1 n}$ therefore reduce to

$$
\begin{aligned}
& a_{0 n}=\delta_{n, 0} \\
& \beta_{j 0}^{\ell}=\left(1-\delta_{\ell, 0} \delta_{j, 0}\right) \frac{\left\langle f_{j} \mid u_{\ell}\right\rangle}{\lambda_{j}+\mathrm{i} \ell \omega_{o}}, \\
& a_{1 n}=-\sum_{\ell=-\infty}^{\infty}\left(1-\delta_{\ell, 0} \delta_{n, 0}\right) \frac{\left\langle f_{n} \mid u_{\ell}\right\rangle}{\lambda_{n}+\mathrm{i} \ell \omega_{o}},
\end{aligned}
$$

where $\left\langle f_{j}\left|u_{\ell}\right| f_{0}\right\rangle=\left\langle f_{j}\left|u_{\ell}\right| 1\right\rangle=\left\langle f_{j} \mid u_{\ell}\right\rangle$ has been used. Hence, (3.18) becomes

$$
\frac{\mathrm{d} M_{1}}{\mathrm{~d} t}=P e^{\prime} \sum_{k=-\infty}^{\infty}\left\langle u_{k} \mid 1\right\rangle \mathrm{e}^{-\mathrm{i} k \omega_{o} t},
$$




$$
\begin{aligned}
M_{1}= & P e ́ \sum_{\ell=-\infty}^{\infty}\left\langle u_{\ell} \mid 1\right\rangle \frac{1-\mathrm{e}^{-\mathrm{i} \ell \omega_{o} t}}{\mathrm{i} \ell \omega_{o}} \\
= & P e ́\left\{\left\langle u_{0} \mid 1\right\rangle t+\sum_{\ell=-\infty}^{\infty}\left(1-\delta_{\ell, 0}\right)\left\langle 1 \mid u_{\ell}\right\rangle \frac{\mathrm{e}^{\mathrm{i} \ell \omega_{o} t}-1}{\mathrm{i} \ell \omega_{o}}\right\}, \\
\frac{1}{2} \frac{\mathrm{d} M_{2}}{\mathrm{~d} t}= & 1+P^{\prime} e^{2}\left\{\sum_{\ell=-\infty}^{\infty}\left\langle u_{\ell} \mid 1\right\rangle\left\langle u_{0} \mid 1\right\rangle t \mathrm{e}^{-\mathrm{i} \ell \omega_{o} t}+\sum_{m=0}^{\infty} \sum_{\ell=-\infty}^{\infty} \sum_{k=-\infty}^{\infty} \frac{\left\langle u_{k} \mid f_{m}\right\rangle\left\langle f_{m} \mid u_{\ell}\right\rangle}{\lambda_{m}+\mathrm{i} \ell \omega_{o}}\right. \\
& \left.\times\left(1-\delta_{m, 0} \delta_{\ell, 0}\right)\left[\mathrm{e}^{\mathrm{i} \ell \omega_{o} t}-\mathrm{e}^{-\lambda m t}\right] \mathrm{e}^{-\mathrm{i} k \omega_{o} t}\right\},
\end{aligned}
$$

and after a final index change of $m$ to $n$ we arrive at

$$
D_{e f f}(t)=1+P e^{2} \sum_{n=1}^{\infty} \sum_{\ell=-\infty}^{\infty} \sum_{k=-\infty}^{\infty} \frac{\left\langle u_{k} \mid f_{n}\right\rangle\left\langle f_{n} \mid u_{\ell}\right\rangle}{\lambda_{n}+\mathrm{i} \ell \omega_{o}}\left[\mathrm{e}^{\mathrm{i}(\ell-k) \omega_{o} t}-\mathrm{e}^{-\left(\lambda_{n}+\mathrm{i} k \omega_{o}\right) t}\right] .
$$

This is the main theoretical result of our work: a closed expression for the transient Taylor-Aris dispersion $D_{\text {eff }}(t)$ for any given time-dependent, axial flow field $u\left(\boldsymbol{r}_{\perp}, t\right)$ in the case of complete transverse diffusion. The flow frequencies $\ell \omega_{o}$ and solute diffusion relaxation rates $\lambda_{n}$ appears explicitly, while the momentum relaxation rates $\alpha_{f l}$ are implicit in $\left\langle f_{n} \mid u_{\ell}\right\rangle$. The result (3.22), which generalizes previous steady and single-frequency results, is particularly relevant in the field of microfluidics characterized by laminar flow in channels of small cross-sectional dimensions compared with the channel lengths.

The time-averaged diffusivity $D_{e f f}^{a v r}(t)$ over one oscillation period $\tau_{0}=2 \pi / \omega_{o}$ is

$$
\begin{aligned}
D_{e f f}^{a v r}(t)= & \frac{1}{\tau_{0}} \int_{t}^{t+\tau_{0}} D_{e f f}(t) \mathrm{d} t \\
= & 1+P e^{2} \sum_{n=1}^{\infty} \sum_{\ell=-\infty}^{\infty} \sum_{k=-\infty}^{\infty} \frac{\left\langle u_{k} \mid f_{n}\right\rangle\left\langle f_{n} \mid u_{\ell}\right\rangle}{\lambda_{n}+\mathrm{i} \ell \omega_{o}} \\
& \times\left[\delta_{\ell, k}-\frac{1-\mathrm{e}^{-\lambda_{n} \tau_{0}}}{\left(\lambda_{n}+\mathrm{i} k \omega_{o}\right) \tau_{0}} \mathrm{e}^{-\left(\lambda_{n}+\mathrm{i} k \omega_{o}\right) t}\right],
\end{aligned}
$$

which in the long-time limit $t \gg 1 / \lambda_{1}$ reduces to

$$
D_{e f f}^{a v r}(\infty)=1+P e^{2} \sum_{n=1}^{\infty}\left[\frac{\left|\left\langle u_{0} \mid f_{n}\right\rangle\right|^{2}}{\lambda_{n}}+\sum_{\ell=1}^{\infty} \frac{2 \lambda_{n}\left|\left\langle u_{\ell} \mid f_{n}\right\rangle\right|^{2}}{\lambda_{n}^{2}+\ell^{2} \omega_{o}^{2}}\right] .
$$

Note that the $\ell$-sum only runs over positive integers and not as previously over all integers.

Finally, in the case of a steady flow given by

$$
\left|u_{\ell}\right\rangle=\delta_{\ell, 0}\left|u_{0}\right\rangle
$$

expression (3.22) for the effective diffusivity reduces to

$$
D_{e f f}^{\text {steady }}(t)=1+P e^{2} \sum_{n=1}^{\infty} \frac{\left|\left\langle u_{0} \mid f_{n}\right\rangle\right|^{2}}{\lambda_{n}}\left(1-\mathrm{e}^{-\lambda_{n} t}\right),
$$

and hence we recover the steady-flow, transient-solute result of Barton (1983). 


\section{General aspects of dispersion and relaxation rates}

Our main result (3.22) implies directly that for all time-dependent flows the effective diffusivity depends on the velocity squared, $D_{\text {eff }} \propto P^{\prime} e^{2} \propto U_{o}^{2}$. However, the specific form of $D_{\text {eff }}$ depends on the magnitude of the amplitude $\left\|u_{\ell}\right\|$, of a given velocity component with frequency $\ell \omega_{o}$, relative to the amplitude $\left\|u_{0}\right\|$ of the steady component.

For small oscillation amplitudes $\left\|u_{\ell}\right\| \ll\left\|u_{0}\right\|$, the velocity field remains unidirectional, but its magnitude, and hence the shear gradients in the velocity, oscillates with frequency $\ell \omega_{o}$ around the steady value. Consequently, $D_{\text {eff }}(t)$ oscillates with frequency $\ell \omega_{o}$ around its time-averaged value $D_{e f f}^{a v r}(\infty)$. For sufficiently large amplitudes $\left\|u_{\ell}\right\| \gg\left\|u_{0}\right\|$ (the exact limit depends on the channel cross-section geometry), the direction of the velocity field changes sign with frequency $2 \ell \omega_{o}$. As a result, due to the terms with $k=-\ell$ in $(3.23 b), D_{\text {eff }}(t)$ also oscillates with the double frequency $2 \ell \omega_{o}$. Moreover, because this frequency doubling ensures a non-zero, time-averaged effective diffusivity $D_{e f f}^{a v r} \propto\left\|u_{\ell}\right\|^{2} P e^{2}, D_{e f f}^{a v r}$ increases above $D_{\text {eff }}^{\text {steady }}$. This reflects that now $\left\|u_{\ell}\right\| U_{o}$ and not $U_{o}$ should be chosen as $U_{\text {char }}$ in (2.4), thus quantifying the observations made in the chemical engineering community that pulsating flows lead to increased mass transfer (Taylor \& Leonard 1965).

By definition, the variance of the solute distribution $\mu_{2}(t)$ is positive at all times, but this does not imply that the differential variance $D_{e f f}(t)=(1 / 2)\left(\mathrm{d} \mu_{2} / \mathrm{d} t\right)$ also remains positive; in fact, negative values of $D_{\text {eff }}(t)$ are often encountered. In general, for short times $t \ll 1 / \lambda_{1}, 1 /\left(2 \ell_{\max } \omega_{o}\right)$, we find $D_{\text {eff }}(t)=1+t P e^{2} \sum_{n=1}^{\infty}\left|\left\langle f_{n} u(0)\right\rangle\right|^{2}>0$, see Appendix, while for steady-flow oscillations of large amplitudes $\left\|u_{\ell}\right\| \gg\left\|u_{0}\right\|$ the transient contraction of the solute concentration field associated with each reversal of the solvent flow direction leads to negative values of $D_{\text {eff }}(t)$. The cross-over point to negative values of $D_{\text {eff }}(t)$ depends on the relative amplitudes of all components of the velocity field, and is therefore not easily estimated in the general case. However, for the simple case of a single-frequency flow the cross-over point can be identified from (3.24). The time-averaged (positive) level is set by the diagonal terms $\ell=k$ for $-1,0$ and 1 in the $n$-sum given by $\left|\left\langle u_{0} f_{n}\right\rangle\right|^{2} / \lambda_{n}+\left|\left\langle u_{1} f_{n}\right\rangle\right|^{2} 2 \lambda_{n} /\left(\lambda_{n}^{2}+\omega_{o}^{2}\right)$, while the cross-terms $\ell=-k$ for -1 and 1 gives the oscillating terms $2 \operatorname{Re}\left[\left(\left\langle u_{1} \mid f_{n}\right\rangle \mathrm{e}^{\mathrm{i} \omega_{o} t}\right)^{2} /\left(\lambda_{1}+\mathrm{i} \omega_{o}\right)\right]$. For the dominant $n=1$ term at the intermediate frequency $\lambda_{1} \ll \omega_{o} \lesssim S c$, we can neglect $\lambda_{1}$ in the denominators, and since $W o=\sqrt{\omega_{o} / S c} \lesssim 1$ we have $\left\langle u_{1} \mid f_{n}\right\rangle \approx\left\langle u_{0} \mid f_{n}\right\rangle\left\|u_{1}\right\| /\left\|u_{0}\right\|$, which results in $D_{\text {eff }} \propto 1+2\left(\left\|u_{1}\right\|^{2} /\left\|u_{0}\right\|^{2}\right)\left(\lambda_{1} / \omega_{o}\right)\left[\left(\lambda_{1} / \omega_{o}\right)+\sin \left(2 \omega_{o} t+2 \phi_{0}\right)\right]$, where the phase $\phi_{0}$ is given by $\left\langle u_{1} \mid f_{n}\right\rangle=\left|\left\langle u_{1} f_{n}\right\rangle\right| \mathrm{e}^{\mathrm{i} \phi_{0}}$. Negative values of $D_{\text {eff }}(t)$ are therefore expected for $\left\|u_{1}\right\|>\left\|u_{0}\right\| \sqrt{\omega_{o} /\left(2 \lambda_{1}\right)}$.

In the absence of a steady component in the velocity field, $u_{0}=0$, the frequency doubling is always present. Thus, for purely oscillating flows one finds a shearenhanced dispersion above molecular diffusion, even though there is no net flow.

General conclusions for the shape dependence of $D_{\text {eff }}$ may be obtained by applying random matrix theory (Mehta 2004) to the geometry-dependent inner product/eigenvalue expressions (3.22), (3.24) and (3.26) following the analysis of the shape-dependent quantum transport through quantum dots (Bruus \& Stone 1994). For a non-integrable system the values of the inner products in (3.26) can be regarded as a random distribution with an average of the order unity. We therefore expect the sum to be dominated by the lowest eigenvalues, for which $\lambda_{n} \propto 1 / R^{2}$, where $R$ is the aspect ratio of the characteristic length scales of the two cross-section directions $(R \geqslant 1)$, which for a rectangle is the wide 'width' divided by the short 'height'. Consequently 
$\left(D_{e f f}^{\text {steady }}-1\right) \propto P e^{-2} R^{2}$, indicating that the dominating length scale no longer is the short 'height' but the wide 'width'. This is in agreement with the analysis of the shallow, slowly varying cross-sections studied by Ajdari et al. (2006) and Dutta et al. (2006).

The solute dispersion can be calculated from (3.22), if the Fourier series of the flow velocity is known, e.g. the single-frequency flow in a Newtonian fluids (Womersley 1955) or steady non-Newtonian fluid flow (Fan \& Wang 1966). However, in the rest of the paper we restrict the application of our theory to the case of incompressible Newtonian fluids of kinematic viscosity (or momentum diffusivity) $v$ in the laminar regime which are governed by the time-dependent Stokes equation,

$$
\partial_{t} u\left(\boldsymbol{r}_{\perp}, t\right)=S c\left[\nabla_{\perp}^{2} u\left(\boldsymbol{r}_{\perp}, t\right)+\frac{1}{\mathscr{L}} \Delta p(t)\right] .
$$

Here, $\Delta p(t)$ is the time-dependent pressure drop along the channel of length $\mathscr{L}$, resolved by the components $\varepsilon_{\ell} \Delta p \mathrm{e}^{\mathrm{i} \ell \omega_{o} t}$ ( $\varepsilon_{\ell}$ is a dimensionless amplitude) with the dimensionless base frequency $\omega_{o}=W o^{2} S c$, and the dimension-full pressure has been normalized by the shear-induced pressure $P_{o}=\left(\eta U_{o}\right) / L_{o}$. The Schmidt number $S c$ appears since the time scale has been chosen to be the transverse mass diffusion time and not the momentum diffusion time. The linearity of this equation allows us to solve the flow problem analytically and thereby obtain the Fourier coefficients $u_{\ell}$ and the momentum diffusion rate $\alpha_{f l}$. The dispersion $D_{\text {eff }}(t)$ depends on $\alpha_{f l}$, and thus on Wo by (2.8), implicitly through the velocity components $u_{\ell}$.

The effective diffusivity varies greatly depending on the system parameters. Below, we provide explicit estimates of the relaxation rates of the solute and fluid momentum, by analysing specific time-dependent systems of increasing complexity through the addition of more time scales to both motion of the solvent and diffusion of the solute. We interpret the results for $D_{\text {eff }}$ in terms of the relaxation rates and the flow frequencies in agreement with the general considerations just outlined.

\section{Multiple-frequency flow in one-length-scale cross-sections}

We begin by analysing channel cross-sections with only one inherent length scale, such as the circular tube or the infinite parallel-plate slit, and the associated time scale for transverse solute diffusion. All results presented in this section are computed for the circular cross-section, but qualitatively they apply to other singlelength cross-sections. We consider a circular tube of unit radius (the radius being $a=L_{o}$ in dimension-full coordinates) and calculate the velocity field from the Stokes equation, (4.1). Using cylindrical coordinates and a generalized wavenumber $k_{\ell}$, the velocity component $u_{\ell}$ fulfilling the boundary conditions $\left.u\right|_{r=1}=0$ and $\left.\partial_{r} u\right|_{r=0}=0$ is (Womersley 1955)

$$
\begin{aligned}
k_{\ell} & =k_{\ell}(W o)=\sqrt{-\mathrm{i} \ell W o^{2}}=\sqrt{-\mathrm{i} \ell \omega_{o} / S c}, \\
\left|u_{\ell}\right\rangle & =\varepsilon_{\ell} \frac{8}{k_{\ell}^{2}}\left[\frac{J_{0}\left(k_{\ell} r\right)}{J_{0}\left(k_{\ell}\right)}-1\right],
\end{aligned}
$$

where the steady-state Poiseuille solution is $u_{0}(r)=\lim _{\ell \rightarrow 0} u_{\ell}(r)=\varepsilon_{0} 2\left(1-r^{2}\right)$. The velocity scale is taken to be the average steady state velocity $U_{o}=\Delta p a^{2} /(8 \eta \mathscr{L})$ for $\varepsilon_{0}=1$. The eigenfunctions and eigenvalues corresponding to (3.4) are

$$
\left|f_{n}\right\rangle=\delta_{n, 0}+\left(1-\delta_{n, 0}\right) \frac{J_{0}\left(\xi_{1, n} r\right)}{J_{0}\left(\xi_{1, n}\right)},
$$




$$
\lambda_{n}=\left(1-\delta_{n, 0}\right) \xi_{1, n}^{2},
$$

where $J_{s}(x)$ is the Bessel function of the first kind of order $s$, and $\xi_{s, n}$ is the $n$th root of $J_{s}(x)$. For cylindrical coordinates (axisymmetric case) $\langle f \mid g\rangle=\int_{0}^{1} \mathrm{~d} r 2 r f^{*}(r) g(r)$, so

$$
\left\langle f_{n} \mid u_{\ell}\right\rangle=-\varepsilon_{\ell} \frac{16}{\left(\xi_{1, n}^{2}-k_{\ell}^{2}\right) k_{\ell}} \frac{J_{1}\left(k_{\ell}\right)}{J_{0}\left(k_{\ell}\right)} \quad \text { for } n>0,
$$

and $\left\langle f_{n} \mid u_{0}\right\rangle=\lim _{\ell \rightarrow 0}\left\langle f_{n} \mid u_{\ell}\right\rangle=-8 / \xi_{1, n}^{2}$ for the steady term. Using these $\left\langle f_{n} \mid u_{\ell}\right\rangle$ in (3.22) allows us to calculate $D_{\text {eff }}(t)$ for an unsteady flow, and by inserting them in (3.26) we recover $D_{\text {eff }}^{\text {steady }}(t)=1+P e^{-2}\left[(1 / 48)-\sum_{n=1}^{\infty}\left(64 / \xi_{1, n}^{6}\right) \exp \left(-\xi_{1, n}^{2} t\right)\right]$, the classic result for a transient solute concentration in a steady flow obtained by Barton (1983).

The fluid momentum equilibration rate for the circular cross-section is $\alpha_{f l}=S c \xi_{0,1}^{2}$, so the generalized wavenumber $k_{\ell}=\sqrt{-\mathrm{i} \ell W o^{2}}=\xi_{0,1} \sqrt{-\mathrm{i} \ell \omega_{o} / \alpha_{f l}}$ and the overlap integrals (5.3) depend explicitly on the fluid inertia through the ratio of the driving frequency $\omega_{o}$ to the fluid momentum equilibration rate $\alpha_{f l}$.

\subsection{A steady-plus-one-frequency flow}

We consider now the simple case of a steady flow of fixed amplitude $\varepsilon_{0}=1$ with the addition of one oscillating component of variable amplitude $\varepsilon_{1}$. The dispersion in this particular flow has previously been studied to various levels of detail (Aris 1960; Chatwin 1975; Mukherjee \& Mazumder 1988). Our theory both recovers, quantifies and provides insight to the underlying physical mechanisms encountered in these previous studies.

$D_{\text {eff }}(t)$ transiently builds towards a steady-oscillation level on the time scale $1 / \lambda_{1}=1 / \xi_{1,1}^{2}=0.068$, as seen in the example figure $1(a)$ (black line) for $\varepsilon_{1}=0.05$, $P e ́=20, S c=1000, \omega_{o}=200$ and $W o=\sqrt{\omega_{o} / S c}=0.447$. As expected from the general observations in $\S 4, D_{\text {eff }}(t)$ oscillates around $D_{\text {eff }}^{\text {avr }}(t) \approx D_{\text {eff }}^{\text {steady }}(t)$ for $\varepsilon_{1}^{2} \ll \varepsilon_{0}^{2}=1$ (grey line). The inset shows increasing $D_{e f f}^{a v r}(t)$ for growing oscillation amplitude, $\varepsilon_{1}=0,3$ and 12 , where $D_{\text {eff }}^{\text {avr }}$ changes from following $D_{\text {eff }}$ fairly closely at $\varepsilon_{1}=3$ to being substantially enhanced for $\varepsilon_{1}=12$. In figure $1(b)$ we zoom in on the two grey zones of $(a)$ to show the excellent agreement between our theory for $D_{\text {eff }}(t)-D_{\text {eff }}^{\text {steady }}(t)$ (full line), numerics (circles, see Appendix for details), and the single-frequency theory of Mukherjee \& Mazumder (1988) (stars).

Extending the physical analysis provided by Mukherjee \& Mazumder (1988), we have augmented $\varepsilon_{1}$ by a factor of 1000 to the value 50 in figure $1(c)$, and the normalized plot $D_{\text {eff }}(t) / D_{\text {eff }}^{\text {avr }}(\infty)$ illustrates that the oscillation-induced enhancement of $D_{\text {eff }}^{a v r}(t)$ shown in the inset of $(a)$ is accompanied by both a frequency doubling in, and negative values of, $D_{\text {eff }}(t)$ as discussed in $\S 4$. The onset of this nonlinearity in the dispersion is further investigated in figure $1(d)$, where $D_{e f f}^{\text {avr }}(\infty) / D_{\text {eff }}^{\text {steady }}(\infty)$ is plotted versus the oscillation amplitude $\varepsilon_{1}$ : a cross-over from the linear regime, where $D_{\text {eff }}^{\text {aur }}(\infty) / D_{\text {eff }}^{\text {steady }}(\infty) \approx 1$, to the nonlinear regime, where $D_{\text {eff }}^{\text {avr }}(\infty) / D_{\text {eff }}^{\text {steady }}(\infty) \propto \varepsilon_{1}^{2}$, happens at a frequency-dependent critical value $\varepsilon_{1}^{c}$. We estimate $\varepsilon_{1}^{c}$ in the lowfrequency limit $W o \lesssim \xi_{0,1} \approx 2.40$ from (3.24) using that in this case $\left\langle u_{1} \mid f_{n}\right\rangle \approx\left\langle u_{0} \mid f_{n}\right\rangle$, and since

$$
\varepsilon_{\ell}=\delta_{\ell, 0}+\varepsilon_{1}\left(\delta_{\ell,-1}+\delta_{\ell, 1}\right),
$$




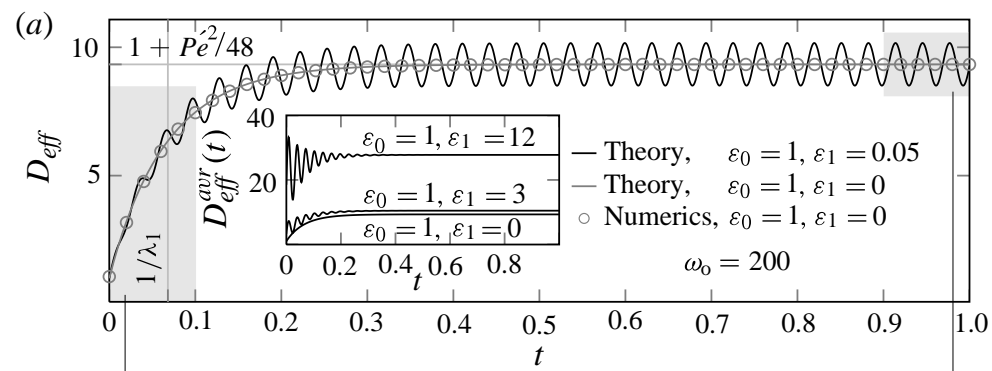

(b)

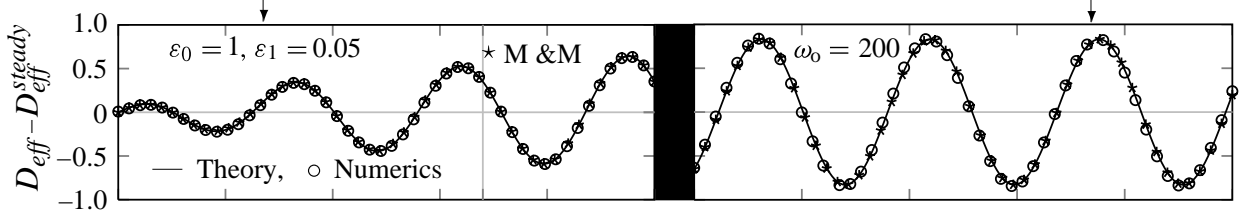

(c)

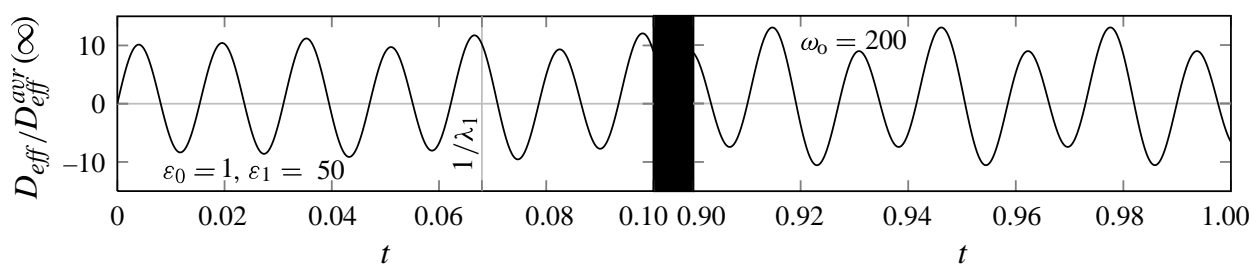

$(d)$

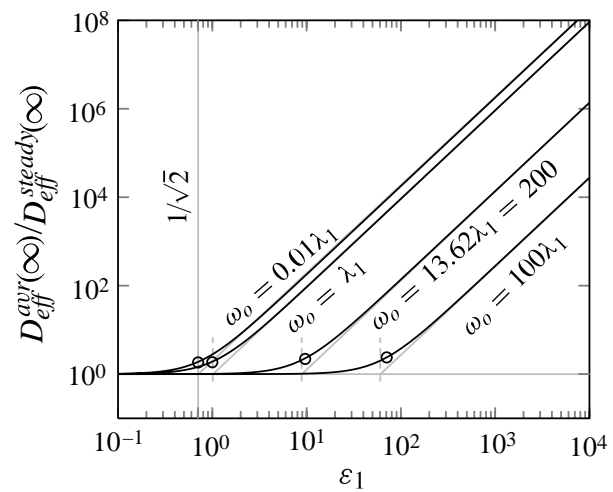

(e)

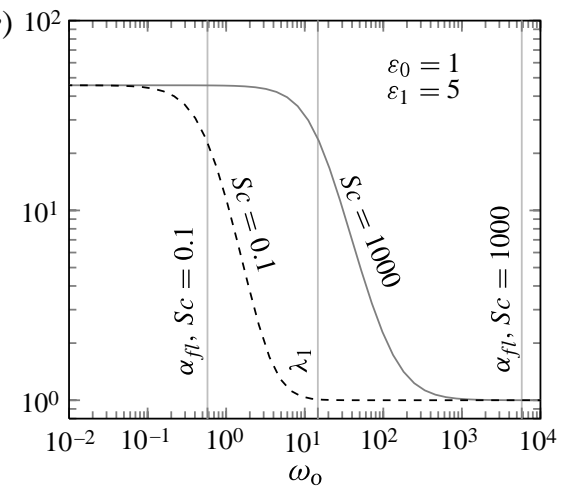

FIGURE 1. $D_{\text {eff }}(t)$ calculated by (3.22) in a tube of circular cross-section for a harmonically oscillating flow of frequency $\omega_{o}$ superimposed on a steady flow, $P e ́=20$. (a) $D_{\text {eff }}(t)$ for $\omega_{0}=200, W o=0.447$ and velocity amplitudes $\varepsilon_{1}=0.05 \ll \varepsilon_{0}=1$ (black line) oscillating around $D_{\text {eff }}^{\text {steady }}(t)$ determined by theory (3.26) (grey line) and by direct numerical simulation (grey circles). The inset shows $D_{e f f}^{\text {avr }}(t)$ for $\varepsilon_{1}=0,3$ and 12, (3.23b). (b) Zoom-in on $D_{\text {eff }}(t)$ for the initial transient period $0<t<0.1$ (left) and the steady regime $0.9<t<1$ (right) showing agreement between our theory (black line), our numerics (circles) and the theory of Mukherjee \& Mazumder (1988) (M \& M). (c) Normalized effective diffusivity $D_{\text {eff }}(t) / D_{\text {eff }}^{\text {avr }}(\infty)$ showing frequency doubling; parameters as in $(b)$ except now $\varepsilon_{1}=50$. (d) $D_{\text {eff }}^{\text {avr }}(\infty) / D_{\text {eff }}^{\text {steady }}(\infty)$, (5.5), versus $\varepsilon_{1}$ for four different frequencies $\omega_{o}$. The cross-over point $\varepsilon_{1}^{c}$ (open circles) from the linear regime $\left(D_{\text {eff }}^{\text {avr }} / D_{\text {eff }}^{\text {steady }} \approx 1\right)$ to the nonlinear one $\left(D_{\text {eff }}^{\text {avr }} / D_{\text {eff }}^{\text {steady }} \propto \varepsilon_{1}^{2}\right)$ increases for increasing $\omega_{o}$, as described by (5.6). (e) $D_{\text {eff }}^{\text {avr }}(\infty) / D_{\text {eff }}^{\text {steady }}(\infty)$ versus $\omega_{o}$ for two fluids. For $S c=0.1$ (gas-like, dashed line) it decreases to 1 once $\omega_{o}>\alpha_{f l}$ (solvent-momentum-diffusion limited), while for $S c=1000$ (water-like, grey line), it decreases to 1 when $\omega_{o}>\lambda_{1}$ (solute-diffusion limited). 
it follows that

$$
D_{e f f}^{a v r}(\infty) \approx 1+P e^{2} \sum_{n=1}^{\infty}\left[1+\frac{2 \varepsilon_{1}^{2}}{1+\left(\omega_{o} / \lambda_{n}\right)^{2}}\right] \frac{\left|\left\langle u_{0} \mid f_{n}\right\rangle\right|^{2}}{\lambda_{n}} .
$$

Thus, the cross-over to period-doubled behaviour (dominance of the $\varepsilon_{1}^{2}$ term) happens when $\varepsilon_{1}$ equals

$$
\varepsilon_{1}^{c}=\frac{1}{\sqrt{2}} \sqrt{1+\frac{\omega_{o}^{2}}{\lambda_{1}^{2}}}= \begin{cases}\frac{1}{\sqrt{2}} & \text { for } \omega_{o} \ll \lambda_{1}, \\ \frac{\omega_{o}}{\sqrt{2} \lambda_{1}} & \text { for } \omega_{o} \gg \lambda_{1} .\end{cases}
$$

When $\omega_{o} \ll \lambda_{1}$, the solute fully equilibrates by diffusion $\left(\lambda_{1}\right.$ is the solute diffusion equilibration rate) and thereby exploits all velocity gradients, so the cross-over to frequency-doubled behaviour happens as soon as the amplitude of the sinusoidal part of the velocity field exceeds that of the steady component, i.e. at the r.m.s. value $\varepsilon_{1}^{c}=1 / \sqrt{2}$ for $\varepsilon_{0}=1$. In contrast, for $\omega_{o} \gg \lambda_{1}$ the solute diffusion cannot fully follow the solvent oscillations, and only by increasing the amplitude significantly will the oscillation component contribute to $D_{\text {eff }}$, and as a consequence the cross-over amplitude scales as $\varepsilon_{1}^{c} \propto \omega_{o} / \lambda_{1}$. In figure $1(d)$ is shown that the estimates of (5.6) are correct. This result further agrees with, and quantifies, the observations of Chatwin (1975) and Watson (1983), but disagrees with Aris (1960), who based on cases with $\varepsilon_{1} \leqslant 1$ predicted that the pulsatile contribution to $D_{e f f}^{a v r}(\infty)$ is less than $1 \%$, which is clearly incorrect for large values of $\varepsilon_{1}$. For $\varepsilon_{1}>\varepsilon_{1}^{c}$ we have $\left(D_{\text {eff }}^{a v r}-1\right) \propto \varepsilon_{1}^{2} P e^{2}$ signalling the change of characteristic velocity scale discussed in $\S 4$.

In addition to these limitations set by the equilibration of the solute, the dispersion may also be limited by fluid inertia, which similarly to the solute diffusion equilibration rate is characterized by a solvent-momentum equilibration rate $\alpha_{f l}$. All time scales can be resolved by the solute when the driving frequency is much lower than the two equilibration rates, allowing time enough to establish the time-dependent velocity gradients and for the solute to diffuse in them, see figure 1(e). However, $D_{\text {eff }}$ decreases if $\omega_{o}$ exceeds either of these equilibration rates.

The behaviour of $D_{\text {eff }}$ seems quantitatively similar whether the limiting factor is solute diffusion or solvent momentum diffusion, but the underlying physical mechanisms are different. For solute/solvent combinations which are limited by solute diffusion, $\lambda_{1} \ll \alpha_{f l}$ (full grey line in figure $1 e$ ), the solute is constantly oscillating back and forth in addition to the steady motion as caused by the velocity field. This is still the case when $\omega_{o}>\lambda_{1}$, but here the solute only has time to diffuse by the gradients created by the steady velocity; new solute gradients are created by the oscillating part faster than the old gradients are smoothed out by diffusion. This corresponds to the case where the fluid is water.

In the other limit of solute/solvent configuration, $\alpha_{f l} \ll \lambda_{1}$, the limiting factor is the diffusion of fluid momentum (inertia): the fluid momentum does not have time to react to the pressure oscillations at the driving frequency, so the fluid will only be moved by the steady part of the pressure. Thus, only the steady velocity field will shear the solute distribution resulting in the dispersion of only the steady flow. This effect is seen in the dashed black line in figure 1(e), which corresponds to the case where channel radius is the same as for the water case above (thus keeping $\lambda_{1}$ fixed) but the fluid is air (Lide 1995). 


\subsection{A steady-plus-two-frequencies flow}

Through the driving pressure, we now add a second time scale to the flow so it consists of a steady component, and the two frequencies $\omega_{o}$ and $\tilde{\ell} \omega_{o}, \tilde{\ell}$ being an integer. The effects in $D_{\text {eff }}$ of the previous section extend to the second frequency, and we continue to find good agreement between theory and numerics, see figure 2 . We first take $\tilde{\ell}=2$. As expected from the general observations in $\S 4, D_{\text {eff }}(t)$ oscillates around $D_{\text {eff }}^{\text {steady }}$ with the frequencies of the velocity field when the oscillation amplitudes $\varepsilon_{1}$ and $\varepsilon_{2}$ are sufficiently small (limits given below), see figure $2(a)$, while frequency doubling and negative values appear when $\varepsilon_{1}$ and $\varepsilon_{2}$ become large, see figure $2(b)$. As for the single-frequency case, there is substantial increase of $D_{\text {eff }}^{\text {avr }}(\infty) / D_{\text {eff }}^{\text {steady }}(\infty)$ for large values of $\varepsilon_{1}$ and $\varepsilon_{2}$ and the cross-over to nonlinearity for $\tilde{\ell}=2$ may be predicted from simple estimates for $W_{o} \lesssim \xi_{0,1}$ as follows. Since

$$
\varepsilon_{\ell}=\delta_{\ell, 0}+\varepsilon_{1}\left(\delta_{\ell,-1}+\delta_{\ell, 1}\right)+\varepsilon_{2}\left(\delta_{\ell,-2}+\delta_{\ell, 2}\right),
$$

we distinguish between the three regimes $\varepsilon_{2} \ll 1, \varepsilon_{1} \ll 1$ and $\varepsilon_{1}=\varepsilon_{2}$. In the first case we trivially retrieve the result (5.6), while the second case similarly yields

$$
\varepsilon_{2}^{c}=\frac{1}{\sqrt{2}} \sqrt{1+\frac{4 \omega_{o}^{2}}{\lambda_{1}^{2}}}= \begin{cases}\frac{1}{\sqrt{2}} & \text { for } \omega_{o} \ll \lambda_{1}, \\ \frac{\sqrt{2} \omega_{o}}{\lambda_{1}} & \text { for } \omega_{o} \gg \lambda_{1} .\end{cases}
$$

Hence, the frequency doubling for each of the velocity field frequencies is independent, since a significant nonlinear effect is found when either $\varepsilon_{1}>\varepsilon_{1}^{c}$ or $\varepsilon_{2}>\varepsilon_{2}^{c}$. In the third case of $\varepsilon_{1}=\varepsilon_{2}=\varepsilon$, we find

$$
\varepsilon^{c}=\frac{1}{\sqrt{2}} \sqrt{\frac{1+\frac{5 \omega_{o}^{2}}{\lambda_{1}^{2}}+\frac{4 \omega_{0}^{4}}{\lambda_{1}^{4}}}{1+\frac{5 \omega_{o}^{2}}{\lambda_{1}^{2}}}}= \begin{cases}\frac{1}{\sqrt{2}} & \text { for } \omega_{o} \ll \lambda_{1}, \\ \sqrt{\frac{2}{5}} \frac{\omega_{o}}{\lambda_{1}} & \text { for } \omega_{o} \gg \lambda_{1},\end{cases}
$$

where the second thresholds is slightly lower than for the single-frequency (5.6).

Our understanding of the behaviour of $D_{\text {eff }}^{a v r}$ from the involved diffusion processes of fluid momentum and solute, presented in the previous section on single-frequency flow, applies to each of the frequencies of the flow. The addition of a second velocity frequency introduces more shear and hence more gradients to the concentration field and $D_{\text {eff }}^{a v r}$ therefore increases even further when all velocity frequencies can be resolved, see dashed line in figure $2(c)$, where solute diffusion is the limiting process $\left(\lambda_{1} \ll \alpha_{f f}\right)$ and where for clarity the second frequency is $30 \omega_{o}$ instead of the $2 \omega_{o}$. Two distinct decreases are found in this curve: the first is when the solute equilibration rate $\lambda_{1}$ surpasses $30 \omega_{0}$ and the second when $\lambda_{1}$ increases past the base frequency. The decreases arise because the gradients created by $30 \omega_{o}$ and $\omega_{o}$, respectively, can no longer be exploited by the solute. When both frequencies can be resolved $D_{e f f}^{a v r}$ is almost a factor two greater than the single-frequency case of figure 1(e), here reproduced as the grey line, so the second velocity frequency leads to a large enhancement of $D_{\text {eff }}$. Had we chosen $\alpha_{f l} \ll \lambda_{1}$ in the figure, the same decreases would have been observed around $30 \omega_{o} \approx \alpha_{f l}$ and $\omega_{o} \approx \alpha_{f l}$. 
(a)

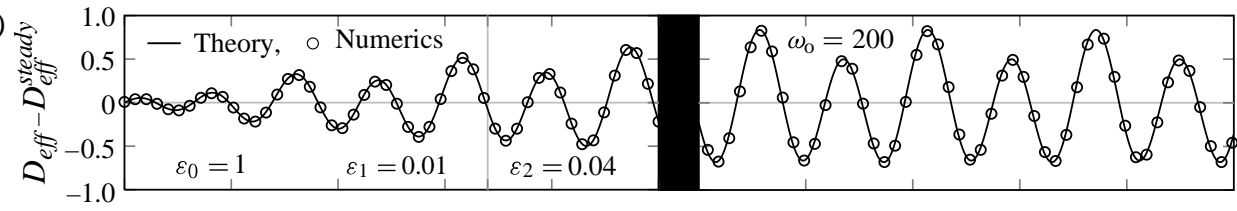

(b)

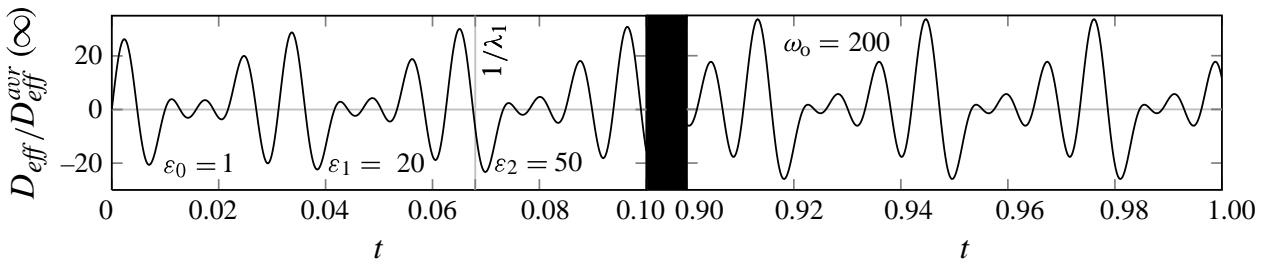

(c)

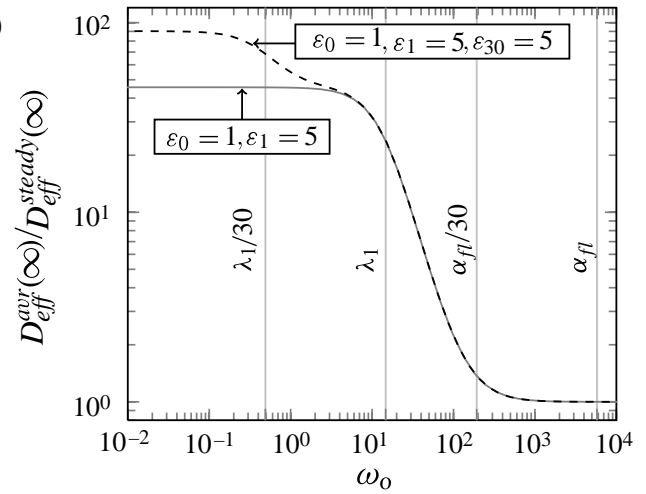

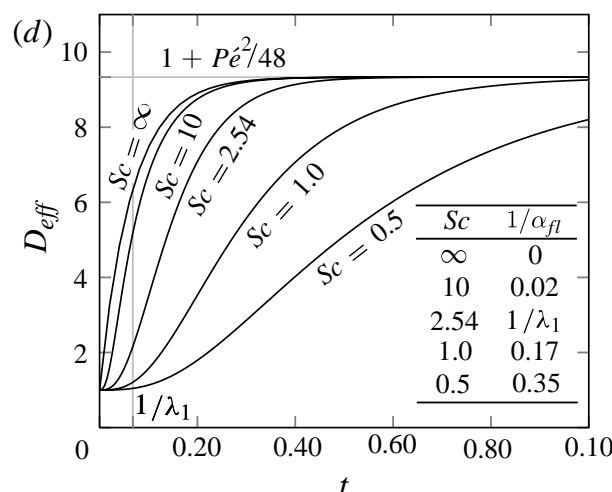

FIgURE 2. Calculated $D_{\text {eff }}(t)$ for a circular cross-section as in figure 1, but with a second oscillating velocity component added. $(a) D_{\text {eff }}(t)-D_{\text {eff }}^{\text {steady }}(t)$ from (3.22) (black line) and numerics (circles) the transient period (left) and the steady regime (right) for small amplitudes, $\varepsilon_{1}=0.01$ and $\varepsilon_{2}=0.04$. (b) As in panel $(a)$, but for large amplitudes, $\varepsilon_{1}=20$ and $\varepsilon_{2}=50$, showing frequency doubling for both $\omega_{o}$ and $2 \omega_{o} .(c) D_{\text {eff }}^{\text {avr }}(\infty) / D_{\text {eff }}^{\text {steady }}(\infty)$ versus $\omega_{o}$ for $S c=10^{3}$ and $\varepsilon_{0}=1, \varepsilon_{1}=\varepsilon_{30}=5$ (dashed curve). For clarity $\tilde{\ell}=30$ is used instead of $\tilde{\ell}=2$ to better illustrate the effects of each frequency. $D_{\text {eff }}^{\text {avr }}(\infty)$ decreases in two steps as $\omega_{o}$ crosses $\lambda_{1} / 30$ and $\lambda_{1}$. The one-frequency case figure $1(e), S c=10^{3}$ (grey curve), is shown for comparison. $(d)$ Startup of tube flow from rest: calculated $D_{\text {eff }}(t)$ versus time for $P e ́=20$. For $S c \lesssim 1$ the dispersion saturates on the solvent acceleration time scale $1 / \alpha_{f l} \approx 1 / S c$, while for $S c \gg 1$ it saturates on the solute diffusion time $1 / \lambda_{1}=1 / \xi_{1,1}^{2} \approx 0.07$. The Fourier series has $T=5$ and contains 1000 terms.

\subsection{Unsteady, unidirectional flow: startup of Poiseuille flow}

In the previous sections we found that fluid inertia limits the solute dispersion because the flow does not equilibrate to a steady state. To further investigate the inertial effects we consider solute in a circular channel where the solvent, after initially being at rest, suddenly at $t=0$ begins to move due to an instantly applied pressure drop along the channel. The analytical solution of this startup of a Poiseuille flow is found in terms of a Fourier-Bessel expansion in the radial coordinate, where each term is multiplied by an exponential time decay of rate $\alpha_{m}$ (Batchelor 1967),

$$
\begin{gathered}
u(r, t)=2\left(r^{2}-1\right)-\sum_{m=1}^{\infty} \frac{16}{\xi_{0, m}^{3}} \frac{J_{0}\left(\xi_{0, m} r\right)}{J_{1}\left(\xi_{0, m}\right)} \mathrm{e}^{-\alpha_{m} t}, \\
\alpha_{m}=S c \xi_{0, m}^{2} \text { for } m=1,2,3, \ldots
\end{gathered}
$$


The smallest of these inertial decay rates, $\alpha_{1}=\xi_{0,1}^{2} S c \approx 5.78 S c=\alpha_{f l}$, sets the characteristic time of the acceleration $1 / \alpha_{f l}$ as in (2.7) and in $\S 5.1$. To align this solution with the developed theory, the temporal functions are written as Fourier series with a base period $T$ much larger than the acceleration time, $T \gg 1 / \alpha_{f l}$. Hence, the flow will reach a steady state significantly faster than the base period $T$, and the approximation will be the correct solution for $0<t<T$. We obtain

$$
\begin{gathered}
u(r, t)=2\left(1-r^{2}\right)-\sum_{m=1}^{\infty} \sum_{\ell=-\infty}^{\infty} \frac{16 J_{0}\left(\xi_{0, m} r\right)}{\xi_{0, m}^{3} J_{1}\left(\xi_{0, m}\right)} A_{\ell m} \mathrm{e}^{\mathrm{i} \ell \omega_{0} t}, \\
A_{\ell m}=\frac{1-\mathrm{e}^{-\alpha_{m} T}}{\alpha_{m} T+\mathrm{i} \ell 2 \pi},
\end{gathered}
$$

where $\omega_{o}=2 \pi / T$ so that $W o=\sqrt{2 \pi /(S c T)}=\xi_{0,1} \sqrt{2 \pi /\left(\alpha_{f f} T\right)}$. The velocity scale is the same as for a steady flow in a circular tube, so the $\ell$ th velocity component $u_{\ell}(r)$ is

$$
u_{\ell}(r)=2\left(1-r^{2}\right) \delta_{\ell, 0}-\sum_{m=1}^{\infty} \frac{16 A_{\ell m} J_{0}\left(\xi_{0, m} r\right)}{\xi_{0, m}^{3} J_{1}\left(\xi_{0, m}\right)} .
$$

Combining this with the eigenvalue solution of (5.2), we find for $n>0$

$$
\left\langle f_{n} \mid u_{\ell}\right\rangle=-\frac{8 \delta_{\ell, 0}}{\xi_{1, n}^{2}}-\sum_{m=1}^{\infty} \frac{32\left(1-\mathrm{e}^{-\alpha_{m} T}\right)}{\xi_{0, m}^{2}\left(\xi_{0, m}^{2}-\xi_{1, n}^{2}\right)\left(\alpha_{m} T+\mathrm{i} \ell 2 \pi\right)} .
$$

The two physical processes of fluid acceleration and solute dispersion happens on the two time scales $1 / \alpha_{f l}$ and $1 / \lambda_{1}$, respectively, but since fluid motion is required to generate the shear needed for the Taylor-Aris dispersion effect, the dispersion is limited by either the solute diffusion, or solvent inertia. In the first case $\left(\alpha_{f l} \gg \lambda_{1}\right)$, the fluid reaches steady state much faster than the solute and the dispersion therefore behaves as in the case of a steady flow. Since $\alpha_{f l} \propto S c$ this can be thought of as having $S c=\infty$, so the only transient behaviour observed is that of the solute diffusion; this is the case of water. For the second case $\left(\alpha_{f l} \propto S c \ll \lambda_{1}\right)$ with slower momentum equilibration rates $0.1<S c<10$ found in gases (Lide 1995), the solute dispersion evolves in the slow increase of the velocity shear gradients, which now dominates the transient behaviour, and $D_{\text {eff }}(t)$ is thus smaller than $D_{\text {eff }}^{\text {steady }}(t)$. These inertial effects are illustrated in figure $2(d)$, where dispersion co-evolves with the transient start-up of a Poiseuille flow in a straight tube at $P e ́=20$, and where the equilibration rates for solute mass diffusion and solvent momentum diffusion are equal for $S c=\xi_{1,1}^{2} / \xi_{0,1}^{2}=2.54$. We take $T=5$, and to diminish unphysical effects of the Gibbs phenomenon close to $t=0$, we include 1000 terms in the Fourier series.

In conclusion, fluid inertia limits fluid shear and hence the solute dispersion. However, given the characteristic time scale $1 / \alpha_{f l} \propto 1 / S c \ll 1 / \lambda_{1}$, inertial effects only become important for Taylor-Aris dispersion in gasses.

\section{Multiple-frequency flow in two-length-scale cross-sections}

We move on to consider the effects of adding a second length scale to the crosssectional geometry. We illustrate this case by use of the rectangle, but the presented findings hold quantitatively for other cross-sections with two length scales, e.g. the ellipse. 


\subsection{The rectangular cross-section}

We denote the height of the channel by $2 a$ and the width by $2 w$, with $w \geqslant a$ so that the aspect ratio $R=w / a$ satisfies $R \geqslant 1$. We take the characteristic length scale to be $L_{o}=a$, so $P e^{\prime}=U_{o} a / D$ and $W o=\sqrt{a^{2} \tilde{\omega}_{o} / \nu}$, and in non-dimensional units we place the rectangular cross-section such that $-R \leqslant y \leqslant R$ and $-1 \leqslant z \leqslant 1$. The analytical velocity field for the steady Poiseuille flow is well known, see e.g. Bruus (2008), and in analogy with $(5.1 b)$ by introducing a generalized wavenumber $q_{j \ell}$, the pulsatile velocity field is found by a trivial extension thereof,

$$
\begin{aligned}
q_{j \ell} & =q_{j \ell}(W o)=\sqrt{\mathrm{i} 4 W o^{2} \ell+j^{2} \pi^{2}} \\
\left|u_{\ell}\right\rangle & =\varepsilon_{\ell}\left(1+\delta_{\ell, 0}\right) \frac{24}{\Gamma} \sum_{j, o d d}^{\infty} \frac{1}{j \pi q_{j \ell}^{2}}\left[1-\frac{\cosh \left(q_{j \ell} \frac{y}{2}\right)}{\cosh \left(q_{j \ell} \frac{R}{2}\right)}\right] \sin \left(j \pi \frac{z+1}{2}\right),
\end{aligned}
$$

where the prefactor $\Gamma$ and the velocity scale $U_{o}$ (chosen as the is the cross-sectional average of the steady flow with $\varepsilon_{0}=1$ ) are given by

$$
\begin{gathered}
\Gamma=\Gamma(R)=1-\sum_{j, \text { odd }}^{\infty} \frac{1}{j^{5}} \frac{192}{\pi^{5}} \frac{1}{R} \tanh \left(j \pi \frac{R}{2}\right), \\
U_{o}=\frac{\Delta p a^{2} \Gamma}{3 \eta \mathscr{L}} .
\end{gathered}
$$

The steady flow profile is retrieved from $(6.1 b)$ by the limit $u_{0}(y, z)=\lim _{\ell \rightarrow 0} u_{\ell}(y, z)$ using $q_{j 0}=j \pi$. Similar to the circular cross-section, the generalized wavenumber $q_{j \ell}$ depends explicitly on the fluid momentum equilibration rate $\alpha_{f l}=\left(1+1 / R^{2}\right)\left(\pi^{2} / 4\right) S c$ since $q_{j \ell}=\pi \sqrt{\mathrm{i} \ell\left(1+1 / R^{2}\right)\left(\omega_{o} / \alpha_{f l}\right)+j^{2}}$. The corresponding basis functions and eigenvalues are

$$
\begin{aligned}
\left|f_{n m}\right\rangle & =\frac{2 \cos \left(n \pi \frac{y+R}{2 R}\right) \cos \left(m \pi \frac{z+1}{2}\right)}{\sqrt{\left(1+\delta_{n, 0}\right)\left(1+\delta_{m, 0}\right)}}, \\
\lambda_{n m} & =\left(\frac{n \pi}{2 R}\right)^{2}+\left(\frac{m \pi}{2}\right)^{2}, \quad n, m=0,1,2, \ldots,
\end{aligned}
$$

which satisfy the requirement $\left|f_{00}\right\rangle=1$ with $\lambda_{00}=0$. The single index $n$ of (5.2) is here changed to the double index $(n, m)$, and $n=0$ to $(n, m)=(0,0)$. The inner products $\left\langle f_{n} \mid u_{\ell}\right\rangle$ of (3.22) become $\left\langle f_{n m} \mid u_{\ell}\right\rangle$ given by

$$
\left\langle f_{n m} \mid u_{\ell}\right\rangle= \begin{cases}\frac{\epsilon_{\ell}\left(1+\delta_{l, 0}\right)}{\sqrt{\left(1+\delta_{n, 0}\right)\left(1+\delta_{m, 0}\right)}} \frac{96 R}{\Gamma \pi^{2}} & \\ \times \sum_{j, o d d}^{\infty} \frac{q_{j l} R \delta_{n, 0}-2 \tanh \left(\frac{q_{j l} R}{2}\right)}{q_{j l}\left(j^{2}-m^{2}\right)\left(n^{2} \pi^{2}+q_{j l}^{2} R^{2}\right)} & n, m \text { even } \\ 0 & \text { otherwise }\end{cases}
$$

and sums $\sum_{n=1}^{\infty}=\sum_{n \neq 0}^{\infty}$ become $\sum_{\{n, m\} \neq\{0,0\}}^{\infty}$. 
The two inherent length scales of this cross-section introduce two diffusion times, one along each coordinate direction, and hence two solute equilibration rates,

$$
\lambda_{20}(R)=\frac{\pi^{2}}{R^{2}} \text { (width) and } \lambda_{02}=\pi^{2} \text { (height). }
$$

We have previously described how the behaviour of $D_{\text {eff }}$ can be understood in terms of the involved diffusion processes of fluid momentum and solute, and it follows directly that while this obviously extends to the present case, the presence of the additional time scales from the second geometric dimension introduces more structure: for each frequency in the fluid velocity field, the solute dispersion will increase if it has time to equilibrate along either of its transverse directions (i.e. if $\omega_{o} \lesssim \lambda_{20}$ or $\left.\omega_{o} \lesssim \lambda_{02}\right)$, with more dispersivity when the frequency allows equilibration along both directions. Thus, for a velocity field with two frequency scales there will be four critical frequencies: the two solute equilibration rates for each of the two fluid time scales. This is illustrated in figure $3(a)$ for the case of $\varepsilon_{0}=\varepsilon_{1}=\varepsilon_{30}=1$ with $P e ́=20$ and $R=100$, and for a solvent/solute composition such that the equilibration rate of the solvent momentum $\alpha_{f l}=\left(1+1 / R^{2}\right)\left(\pi^{2} / 4\right) S c$ is much greater than those of the solute diffusion, $\lambda_{20}$ and $\lambda_{02}$. As function of driving frequency $\omega_{o}, D_{e f f}^{a v r}$ decreases in four steps: the first two and most significant drops happen near $\lambda_{20} / 30$ and $\lambda_{20}$, where the diffusion across the width no longer can exploit the gradients of first the $30 \omega_{o}$-harmonic and then the $\omega_{o}$-harmonic. The last two (minor) drops happen near $\lambda_{02} / 30$ and finally $\lambda_{02}$, where the height diffusion ceases to be able to follow the $30 \omega_{o}$-harmonic and lastly the $\omega_{o}$-harmonic. The substantial increases in time-averaged dispersion found for the two-frequency flow of the previous section is again found in this case: for low frequencies $\omega_{o}$ which allow complete solute equilibration, $D_{e f f}^{a v r}$ is almost a factor of two greater than when only the steady velocity contributes to the solute dispersion $\left(D_{\text {eff }}^{\text {avr }}(\infty)=120.41\right.$ at $\omega_{o}=10^{-8}$ compared with $D_{\text {eff }}^{\text {avr }}(\infty)=60.70$ at $\left.\omega_{o}=10^{4}\right)$. For comparison we have also included the special case of $R=1$, a one-length-scale cross-section, where the two solute diffusion equilibration rates $\lambda_{20}$ and $\lambda_{02}$ are identical, and where we consequently find only two decreasing steps. We have also validated our theory against numerics for the case $R=1$ (details given in Appendix), and find excellent agreement.

Since the solute equilibration rate $\lambda_{20}$ depends on the aspect ratio $R$, (6.5), the same frequency $\omega_{o}$ and velocity component amplitudes $\varepsilon_{\ell}$ will give rise to different behaviours of $D_{\text {eff }}$ in channels of different $R$. This is plotted in figure $3(b)$ for $\omega_{o}=10^{4}$ and $\omega_{o}=10^{-7}$ with the flow chosen as in $(a)$ to be a two-frequency flow with amplitudes $\varepsilon_{0}=\varepsilon_{1}=\varepsilon_{30}=1$. For reference we have also plotted the dependence of $D_{\text {eff }}^{\text {steady }}$ in the limit $R \rightarrow \infty$ where we retrieve the well-known result (Doshi et al. 1978; Chatwin \& Sullivan 1982) $D_{\text {eff }}^{\text {steady }}(\infty)=1+\chi_{\text {rect }} P^{\prime 2}$ with $\chi_{\text {rect }}=2 / 105+11532 \zeta(5)^{2} / \pi^{10}$ where $\zeta(x)=\sum_{j=1}^{\infty} j^{-x}$ is the Riemann zeta function. For sufficiently fast oscillation, here $\omega_{o}=10^{4}$ (lower black line), we have $\omega_{o} \gg \lambda_{02}$, and as in figure $2(b)$ this is too fast for the solute diffusion to follow, and we recover the steady-flow case (triangles) and $D_{\text {eff }}^{\text {avr }}(\infty)=D_{\text {eff }}^{\text {steady }}(\infty)$. Had we chosen larger oscillation amplitudes $\varepsilon_{\ell}$ the nonlinear effect discussed in $\S 4$ would had set in and increased $D_{\text {eff }}^{a v r}$. For the fixed slow oscillation frequency $\omega_{o}=10^{-7}$ (top black

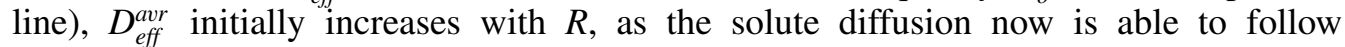
the fluid movement. However, due to the $R$-dependence of $\lambda_{20}$ in (6.5), a point is reached beyond which $\lambda_{20}(R) / 30<\omega_{o}$, and $D_{\text {eff }}^{\text {avr }}$ decreases to a lower value. Later, when $\lambda_{20}(R)$ falls below $\omega_{o}$, a second decrease occurs, after which $D_{\text {eff }}^{a v r}$ settles 

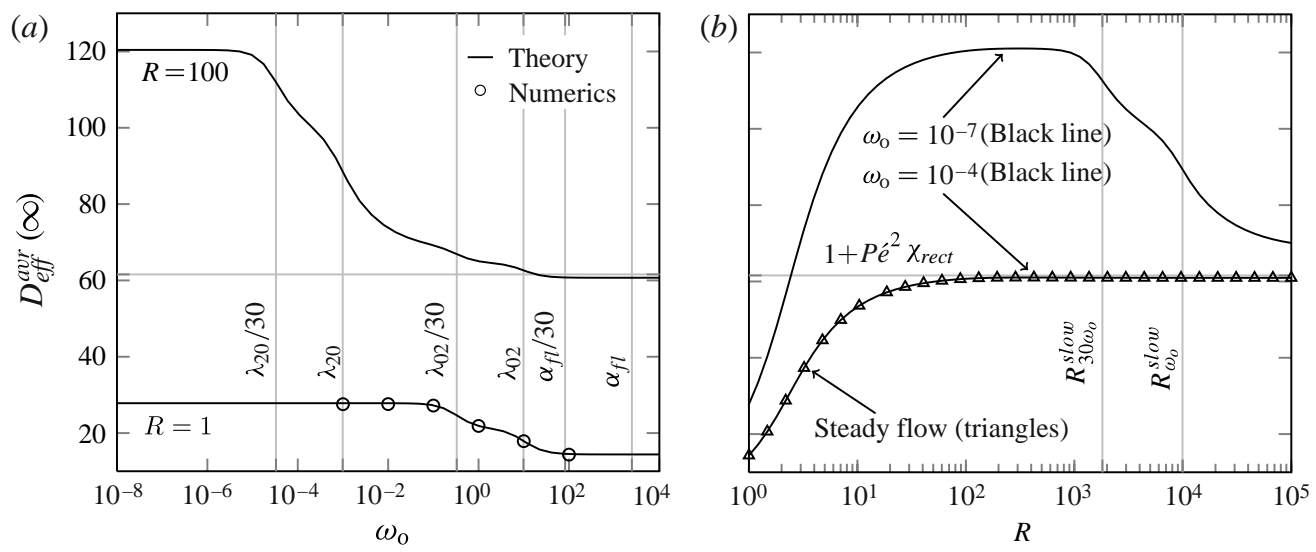

FIGURE 3. $D_{e f f}^{a v r}(\infty)$ for a two-frequency flow $\varepsilon_{0}=\varepsilon_{1}=\varepsilon_{30}=1$ in rectangular channels of aspect ratio $R$. (a) $D_{e f f}^{\text {aur }}(\infty)$ versus base frequency $\omega_{o}$ for $R=1$ and $R=100$ at $P e ́=20$, and with the agreement with numerics (circles) illustrated at $R=1$ (details given in Appendix). As $\omega_{o}$ increases $D_{\text {eff }}^{a v r}$ decreases in four steps, namely when $\omega_{o}$ crosses $\lambda_{20} / 30, \lambda_{20}, \lambda_{02} / 30$, and $\lambda_{02}$ (for $R=100$ ). For $R=1$ there are only two steps, namely when $\omega_{o}$ crosses $\lambda_{02} / 30$ and $\lambda_{02}$ this rate being the same for $R=1$ and $R=100$. $(b) D_{\text {eff }}^{a v r}(\infty)$ versus $R$ for fast oscillation $\left(\omega_{o}=10^{4}\right)$ and steady flow, both recovering the behaviour of figure 1(a), and for slow oscillation $\left(\omega_{o}=10^{-7}\right)$, for which $D_{\text {eff }}(\infty)$ reaches a maximum before decreasing in two steps at $\lambda_{20}(R)=30 \times 10^{-7}$ and $\lambda_{20}(R)=10^{-7}$, marked by the aspect ratios $R_{30 \omega_{o}}^{\text {slow }}$ and $R_{\omega_{o}}^{\text {slow }}$, respectively.

at $D_{\text {eff }}^{\text {steady }}(\infty)=1+\chi_{\text {rect }} P e^{2}$, since none of the added shear of the time-dependent velocity components contribute to $D_{e f f}^{a u r}$.

The highest value of $D_{e f f}^{a v r}$ achievable for a fixed velocity field with fixed frequency $\omega_{o}$ is found in a region with the upper limit set by $3 \ell_{\max } \omega_{o} \approx \lambda_{20}=\pi^{2} / R^{2}$, where $\ell_{\max }$ is the maximum frequency component in the velocity field, and the lower limit is set by the value of $R$ which ensures that the steady part of the velocity field reaches the maximum dispersion of $D_{\text {eff }}^{\text {steady }}(\infty)=1+P e^{2} \chi_{\text {rect }}, R \lesssim 100$.

Finally, a brief discussion is in order regarding the behaviour of $D_{\text {eff }}^{\text {steady }}(\infty)$ in the limit $R \rightarrow \infty$. It is well known that $\chi_{\text {rect }}=2 / 105+11532 \zeta(5)^{2} / \pi^{10} \approx 7.95 \chi_{\text {plate }}$ where $\chi_{\text {plate }}=2 / 105$ is the coefficient for infinite parallel plates (no sidewalls), which might seem to contradict the general scaling of $\left(D_{\text {eff }}-1\right) \propto P e^{2} / R^{2}$ described in $\S 4$. However, the rectangular cross-section is integrable, and the inner products $\left\langle f_{n 0} \mid u_{0}\right\rangle \propto 1 / R$, because as function of the width coordinate $y, u_{0}$ is constant except for the ends covering a fraction $1 / R$ of the width, while $f_{n 0}$ oscillates, see $(6.1 b)$ and $(6.3 a)$. As a result, the factor $R^{2}$ from $1 / \lambda_{n m}$ is cancelled by the factor $1 / R^{2}$ from the inner product, and $\left(D_{e f f}^{\text {steady }}-1\right) \propto P e^{2}$, making the small height the dominant length scale. This explains why in $\chi_{\text {rect }} \approx \chi_{\text {plate }}$ instead of $\chi_{\text {rect }} \approx \chi_{\text {plate }} R^{2}$. Physically, the steady flow in high-aspect-ratio rectangular channels is independent of the channel width except for boundary layers of width unity near the sidewalls, while it remains parabolic in the height. Compared with the infinite parallel plate channel, the sidewalls therefore increase both the mean velocity and the created gradients in the concentration field along the height, causing an increase of $D_{\text {eff }}(\infty)$, which none the less remains on the order of $D_{\text {eff }}(\infty)$ from the parallel plates. For channel 


$\begin{array}{lllcll}R & 2.67 & \varepsilon_{0} & 1.000 & \alpha_{f l} & 2810 \\ P e ́ & 6.92 & \varepsilon_{1} & 0.352 \exp (-2.19 \mathrm{i}) & \omega_{o} & 2.94 \\ W o & 0.05 & \varepsilon_{2} & 0.203 \exp (-2.24 \mathrm{i}) & \lambda_{20} & 1.39 \\ S c & 1000 & \varepsilon_{3} & 0.059 \exp (+1.54 \mathrm{i}) & \lambda_{02} & 9.87\end{array}$

TABLE 1. Dimensionless parameters of the flow generated by the peristaltic pump of Skafte-Pedersen et al. (2009), see $§ 6.2$.
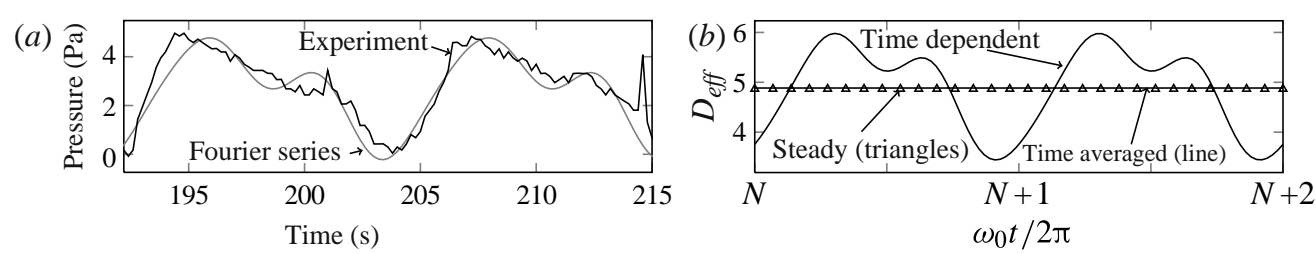

FIGURE 4. (a) Two periods of the measured time-dependent pressure delivered by the peristaltic pump (black) by Skafte-Pedersen et al. (2009) and of the approximate Fourier series $(|\ell| \leqslant 3)$ used in the analysis (grey). (b) Calculated $D_{\text {eff }}(t)$ for the two cycles of the peristaltic pump following period $N=10^{4}$ after the decay of all transients: the Fourier series (black), only the steady component (dashed) and the time-averaged Fourier series (grey).

cross-sections which vary continuously along both cross-section directions (e.g. elliptic), the characteristic length scale of velocity variations is of order $R$, resulting in shear along all of $R$ and the scaling $\left(D_{e f f}^{\text {steady }}-1\right) \propto P e^{2} R^{2}$ as described in $\S 4$.

\subsection{Analysis of a peristaltic micropump}

To illustrate the practical usefulness of the developed theory, we here shortly describe dispersion limiting of a micropump. The pressure delivered by the novel 12-channel, 8-roller, peristaltic pump for microfluidics of Skafte-Pedersen et al. (2009) is pulsating with the operating base frequency $\tilde{\omega}_{o}=0.52 \mathrm{rad} \mathrm{s}^{-1}$, as measured in a rectangular channel of $R=2.67 \quad(a=75 \mu \mathrm{m}, w=200 \mu \mathrm{m})$, and shown by the black line in figure $4(a)$. To analyse the influence of this time-dependent flow on the dispersion of a solute with typical molecular diffusivity $D=10^{-9} \mathrm{~m}^{2} \mathrm{~s}^{-1}$, we approximate the pressure by a Fourier series with $|\ell| \leqslant 3$ (grey line). The discrepancy between the approximation and the actual signal fluctuates faster than the highest harmonic and is not resolved by the solute. The velocity scale $U_{o}$ is taken as the (dominating) steady component and the dimensionless parameters of the system are listed in table 1 .

The pump is designed to have minimal dispersion, which follows from our theory: it is normally operated at $\omega_{o}=2.94$, which is faster than the solute equilibration rate $\lambda_{20}=1.39$ of the width, so only the diffusion in the height direction with $\lambda_{02}=9.87$ is resolved. Furthermore, because the square of all oscillation amplitudes are small, $\left|\varepsilon_{\ell}\right|<0.12$, their contribution to $D_{\text {eff }}$ is minute, and $D_{e f f}^{a v r}(\infty)=1.004 D_{e f f}^{\text {steady }}(\infty)$, see figure $4(b)$. The dispersion may be increased by running the pump at lower frequency, e.g. $D_{\text {eff }}^{\text {avr }}(\infty)=1.17 D_{\text {eff }}^{\text {steady }}(\infty)$ changing $\omega_{o}$ to $0.1 \omega_{o}$, or with higher oscillation amplitudes, $D_{e f f}^{\text {avr }}(\infty)=1.39 D_{\text {eff }}^{\text {steady }}(\infty)$ changing $\varepsilon_{\ell}$ to $10 \varepsilon_{\ell}$ for $\ell \neq 0$. If these changes are made simultaneously, we find $D_{\text {eff }}^{\text {avr }}(\infty)=2.76 D_{\text {eff }}^{\text {steady }}(\infty)$. 


\section{Concluding remarks}

By combining Aris's method of moments and Dirac's bra-ket formalism, we have derived the compact, closed form (3.22) for the transient Taylor-Aris dispersion or effective diffusivity $D_{\text {eff }}(t)$ valid for any time-dependent flow in a long, straight channel of arbitrary but constant cross-section. For a general time-dependent flow and as a function of system parameters, $D_{\text {eff }}(t)$ exhibits a rich and non-trivial structure due to the interplay between internal equilibration rates and external driving frequencies. Our analysis based on (3.22) has led to the following conclusions.

(i) In general, the effective diffusivity is enhanced significantly by those parts of the time-dependent velocity field that have frequencies $\ell \omega_{o}$ lower than the fluid momentum diffusion rate $\alpha_{f l}$ and the solute diffusion rate $\lambda_{1}$, in which case the dispersive effect has sufficient time to fully evolve.

(ii) We have explained why sufficiently large oscillation amplitudes $\left\|u_{\ell}\right\|$ lead to negative values of the instantaneous effective diffusivity $D_{\text {eff }}(t)$. In $\S 4$ an amplitude threshold value for this transition has been derived for a steady-plusone-frequency flow. This threshold depends on the ratio $\sqrt{\omega_{o} /\left(2 \lambda_{1}\right)}$.

(iii) As function of the normalized amplitudes $\varepsilon_{\ell}$ of the oscillatory flow components, scaling laws have been derived for the cross-over $\varepsilon^{c}$ of the time-averaged effective diffusivity $D_{\text {eff }}^{a v}(t)$ from the linear regime of small oscillatory flow components superimposed on a large steady-flow component of normalized amplitude $\varepsilon_{0}$, to the nonlinear, frequency-doubled regime of large oscillation amplitudes. Specific examples are shown in $\S \S 5.1$ and 5.2 for steady-plus-one-frequency and steadyplus-two-frequencies flows.

(iv) Our formulation of $D_{\text {eff }}$ in terms of inner products (overlap integrals) and eigenvalues facilitates general analyses in terms of random matrix theory. As an example of such an analysis we have sketched an explanation for the well-known fact that the relevant Péclet number for the Taylor-Aris dispersion in a channel of rectangular cross-section involves the short height and not as expected on general grounds, the wide width.

(v) For Newtonian solvents we have validated our theory by comparing it with the special cases of dispersion in steady flow and single-frequency pulsating flow treated in the literature, and by direct numerical simulations of single- and multiple-frequency flows in circular (figures $1(b)$ and 2(a)) and rectangular crosssections (figure 3(a)).

(vi) For Newtonian solvents in the cases of one-length scale and steady-plus-onefrequency flow, figure 1(e), one-length scale and steady-plus-two-frequency flow, figure 2(c), and two-length scale and steady-plus-two-frequency flows, figure 3(a), we have characterized the explicit suppression of the oscillatory enhancement of $D_{e f f}^{a v r}\left(\omega_{o}\right)$ each time a driving frequency $\ell \omega_{o}$ becomes larger than the internal diffusion relaxation rate $\lambda_{1}$ or the momentum relaxation rate $\alpha_{f l}$. In particular in figures $1(e)$ and $2(d)$, we found that this suppression is controlled by the solute mass diffusion in liquids and by the solvent momentum diffusion (or inertia) in gasses.

(vii) In $\S 6.2$ we have illustrated the practical usefulness of the developed theory as a design tool for dispersion-control in microfluidic systems.

Our work has resulted in a compact, transparent, and efficient theoretical method for analysing transient Taylor-Aris dispersion in straight channels. Using it, we have 
gained physical insight in, and made explicit predictions of, the effects of general timedependent flows on dispersion. The theory opens up for further generalizations such as including the effects of non-Newtonian solvents and non-trivial channel topologies.

\section{Acknowledgements}

We thank P. Skafte-Pedersen for supplying the experimental pump data of $\S 6.2$, R. Barnkob for assisting with the 64-bit computer used for the numerical part of this work, and H. A. Stone, R. Camassa and R. M. McLaughlin for stimulating discussions on the background literature. This research was supported by grant number 2106-080018 'ProCell', under the Programme Commission on Strategic Growth Technologies, the Danish Agency for Science, Technology and Innovation.

\section{Appendix. Mathematical and numerical details; list of symbols}

A list of quantities used in this work alphabetized by symbol (Latin before Greek) is given in table 2 .

\section{A.1. The Dirac bra-ket notation for spatial dependence}

The basic concept of the bra-ket notation is the inner product of functions, defined by (2.2). It is linear

$$
\left\langle f \mid A_{1} g_{1}+A_{2} g_{2}\right\rangle=A_{1}\left\langle f \mid g_{1}\right\rangle+A_{2}\left\langle f \mid g_{2}\right\rangle,
$$

and given the complex representation (2.6) of the velocity field, we often use that

$$
\begin{gathered}
\left\langle u_{\ell} \mid g\right\rangle=\left\langle g \mid u_{\ell}\right\rangle^{*}=\left\langle g^{*} \mid u_{-\ell}\right\rangle, \\
\left\langle u_{\ell} \mathrm{e}^{\mathrm{i} \ell \omega_{o} t} \mid g\right\rangle=\mathrm{e}^{-\mathrm{i} \ell \omega_{o} t}\left\langle u_{\ell} \mid g\right\rangle .
\end{gathered}
$$

The bra-ket notation is particularly compact when working with basis function expansions. A set of functions $\left|f_{n}\right\rangle, n=0,1,2, \ldots$, is said to form an orthonormal basis when it fulfills the so-called completeness and orthonormal conditions given by

$$
\sum_{n=0}^{\infty}\left|f_{n}\right\rangle\left\langle f_{n}\right|=1 \quad \text { and } \quad\left\langle f_{m} \mid f_{n}\right\rangle=\delta_{m, n},
$$

respectively, where by definition $\delta_{m, n}=1$ for $m=n$, and 0 for $m \neq n$. Any function $|g\rangle$ can formally be expressed by an expansion in this basis as

$$
\begin{gathered}
|g\rangle=\sum_{n=0}^{\infty} a_{n}\left|f_{n}\right\rangle, \\
a_{m}=\left\langle f_{m} \mid g\right\rangle \quad m=0,1,2, \ldots .
\end{gathered}
$$

Here (A $4 b$ ) follows from multiplication of (A $4 a$ ) from the left by $\left\langle f_{m}\right|$ and using (A 3). Since the functions $f$ and $g$ in general depend on time, the inner product may also depend on time. We find that the bra-ket notion allows for a better overview during formal manipulations and lets the underlying structure of the theory stand out more clearly without the clutter of voluminous expressions of integrals. The formalism is particularly convenient for problems involving the Laplace operator, like the present case, because often the corresponding basis functions and eigenvalues are known.

\section{A.2. Method of statistical moments}

The great insight of Aris was to realize that the advection-diffusion problem can be rewritten as a series of equations for statistical moments of the solute distribution, 
Quantity

Cross-sectional part of the $\nabla$ operator

Bra of $\bullet$, and ket of $\bullet$

Complex conjugation

Cross-sectional area

Channel radius/half-height

Concentration field of solute

$p$ th axial moment of concentration

Initial value for $c\left(x, \boldsymbol{r}_{\perp}, t\right)$

Characteristic concentration

Molecular diffusivity

Effective diffusivity

Effective diffusivity, time averaged

Effective diffusivity, steady flow

Cartesian basis vectors

$n$th basis function

Bessel function, first kind of order $s$

Generalized wavenumber, (5.1a)

Channel length

Characteristic length

$p$ th full moment of concentration

Surface normal vector

Pressure

Characteristic pressure

Péclet number

Generalized wavenumber, (6.1a)

Channel aspect ratio

Position vector

Cross-sectional position vector

Schmidt number

Characteristic time

Time

Characteristic velocity

Axial velocity component of solvent

Volume

Velocity field of solvent

Channel half-width

Womersley number

Solute centre of mass

Cylindrical coordinates

Cartesian coordinates

Fluid momentum equilibration rate

Prefactor (6.2a)

Kronecker delta

$\ell$ th velocity component amplitude

Dynamic viscosity

$n$th root of Bessel function $J_{s}$
Symbol

$$
\begin{aligned}
& \nabla_{\perp}=\boldsymbol{e}_{y} \partial_{y}+\boldsymbol{e}_{z} \partial_{z} \\
& \langle\bullet| \text {, and }|\bullet\rangle \\
& * \\
& \mathscr{A} \\
& \text { a } \\
& c \\
& c_{p} \\
& \tilde{c}\left(x, \boldsymbol{r}_{\perp}\right) \\
& C_{o} \\
& D \\
& D_{\text {eff }} \\
& D_{e f f}^{a v r} \\
& D_{\text {eff }}^{\text {steady }} \\
& \boldsymbol{e}_{x}, \boldsymbol{e}_{y}, \boldsymbol{e}_{z} \\
& f_{n} \\
& J_{s} \\
& k_{\ell} \\
& \mathscr{L} \\
& L_{o} \\
& M_{p} \\
& \text { n } \\
& p \\
& P_{o} \\
& P e ́=U_{o} L_{o} / D \\
& q_{j \ell} \\
& R=w / a \\
& \boldsymbol{r} \\
& \boldsymbol{r}_{\perp}=(y, z) \\
& S c=v / D \\
& T_{o} \\
& t \\
& U_{o} \\
& u \\
& \mathscr{V} \\
& v \\
& \text { w } \\
& W o=\sqrt{L_{o}^{2} \tilde{\omega}_{o} / v} \\
& x, r, \varphi \\
& x, y, z \\
& \alpha_{m}, \alpha_{f l} \\
& \Gamma \\
& \delta_{n, m} \\
& \varepsilon_{\ell} \\
& \eta \\
& \xi_{s, n}
\end{aligned}
$$

TABLE 2. (Continued on next page) 
Quantity

Symbol

$n$th diffusion rate eigenvalue

$p$ th moment of concentration about the mean

Kinematic viscosity of solvent

Density of solvent

Diffusivity enhancement

Cross-section

Base frequency, non-dimensionalized

Base frequency, dimension-full

Oscillation period

$$
\begin{gathered}
\lambda_{n} \\
\mu_{p} \\
\nu \\
\rho \\
\chi_{\text {plate }}, \chi_{\text {rect }} \\
\Omega \\
\omega_{o}=W o^{2} S c \\
\tilde{\omega}_{o} \\
\tau_{0}=2 \pi / \omega_{o}
\end{gathered}
$$

TABLE 2. (cntd) List of quantities used in this work alphabetized by symbol (Latin before Greek).

which then can be solved sequentially (Aris 1956). Each additional moment adds new information about the distribution, and some of the lower moments have direct physical interpretations. We here give Aris's definitions and resulting equations of motion for the statistical moments reformulated in the bra-ket notation.

The $p$ th axial moment $c_{p}\left(\boldsymbol{r}_{\perp}, t\right)$ of the solute concentration field $c\left(x, \boldsymbol{r}_{\perp}, t\right)$ and the associated full moment $M_{p}(t)$ are defined by $(2.12 b)$ and $(2.12 a)$ in the main text. Taking the time derivative of these equations and using the advection-diffusion equation (2.9), we obtain the recursive equations of motion (2.13a) and (2.13b) for $c_{p}$ and $M_{p}$ as well as their boundary conditions, (2.14). $M_{0}$ corresponds to the unit norm of $c$, while $M_{1}$ is the time-dependent, axial centre of mass $\bar{x}$ of $c$,

$$
\begin{aligned}
& M_{0}=\langle 1 \mid c\rangle=1, \\
& M_{1}=\langle x \mid c\rangle=\bar{x}(t) .
\end{aligned}
$$

The $p$ th moment $\mu_{p}$ about the centre of mass is defined by

$$
\mu_{p}(t)=\left\langle(x-\bar{x})^{p} \mid c\right\rangle
$$

and by the linearity of the inner product, (A 1 ), we find for $p=0,1$ and 2 ,

$$
\begin{aligned}
& \mu_{0}(t)=\langle 1 \mid c\rangle=1, \\
& \mu_{1}(t)=\langle(x-\bar{x}) \mid c\rangle=0, \\
& \mu_{2}(t)=\left\langle(x-\bar{x})^{2} \mid c\right\rangle=M_{2}(t)-M_{1}(t)^{2} .
\end{aligned}
$$

In particular the time-dependent spatial variance $\mu_{2}$ of the solute concentration is of key interest as it relates the to the solute molecular diffusivity and the effective diffusivity $D_{\text {eff }}$. For a vanishing velocity field, $u=0, \mu_{2}$ relates to the diffusivity $D$ of the solute by $\mathrm{d} \mu_{2} / \mathrm{d} t=2 D$, or in dimensionless form, $\mathrm{d} \mu_{2} / \mathrm{d} t=2$, since:

$$
\begin{aligned}
\frac{\mathrm{d} \mu_{2}}{\mathrm{~d} t} & =\left\langle(x-\bar{x})^{2} \mid \partial_{t} c\right\rangle-2\langle(x-\bar{x}) \mid c\rangle \frac{\mathrm{d} \bar{x}}{\mathrm{~d} t} \\
& =\left\langle(x-\bar{x})^{2} \mid \nabla^{2} c\right\rangle-2 \mu_{1} \frac{\mathrm{d} \bar{x}}{\mathrm{~d} t}=2\langle 1 \mid c\rangle=2,
\end{aligned}
$$

where in the last term we have integrated by parts twice and used the boundary conditions $(2.10 a)$ and $(2.10 c)$. This corresponds to the well-known result for diffusion in one dimension, $(\Delta x)^{2}=2 D t$, with $\mu_{2} \sim(\Delta x)^{2}$. 
When the solvent velocity field $u$ is non-zero the time-dependence of the variance is no longer linear. However, by a traditional generalization (Aris 1956, 1960; Chatwin 1975; Barton 1983; Mukherjee \& Mazumder 1988; Brenner \& Edwards 1993) the dispersion or effective diffusivity $D_{\text {eff }}(t)$ is defined as done in (2.11) in the main text $D_{\text {eff }}(t)=(1 / 2)\left(\mathrm{d} \mu_{2} / \mathrm{d} t\right)=(1 / 2)\left(\mathrm{d} M_{2} / \mathrm{d} t\right)-M_{1}\left(\mathrm{~d} M_{1} / \mathrm{d} t\right)$. Other works have been concerned with the skewness $\mu_{3}$ of the solute distribution (Barton 1983; Camassa et al. 2010). More information about the distribution of $c$ is added with each moment, however, the information contained in each moment remains valid no matter how many moments have been determined.

\section{A.3. The dispersion for short times}

We show here that $D_{e f f}(t)$ is positive for short times $t \ll 1 / \lambda_{1}, 1 /\left(2 \ell_{\max } \omega_{o}\right)$. In this limit we have

$$
\mathrm{e}^{\mathrm{i}(\ell-k) \omega_{o} t}-\mathrm{e}^{-\left(\lambda_{n}+\mathrm{i} k \omega_{o}\right) t}=\left(\lambda_{n}+\mathrm{i} \ell \omega_{o}\right) t+O\left(t^{2}\right),
$$

thus reducing (3.22) for $D_{\text {eff }}(t)$ to

$$
\begin{aligned}
D_{\text {eff }}(t) & \approx 1+P e^{2} \sum_{n=1}^{\infty} \sum_{\ell=-\ell_{\max }}^{\ell_{\max }} \sum_{k=-\ell_{\max }}^{\ell_{\max }}\left\langle u_{k} \mid f_{n}\right\rangle\left\langle f_{n} \mid u_{\ell}\right\rangle t \\
& =1+t \boldsymbol{P}^{-2} \sum_{n=1}^{\infty}\left\langle\sum_{k=-\ell_{\max }}^{\ell_{\max }} u_{k} \mid f_{n}\right\rangle\left\langle f_{n} \mid \sum_{\ell=-\ell_{\max }}^{\ell_{\max }} u_{\ell}\right\rangle \\
& =1+t P e^{-2} \sum_{n=1}^{\infty}\left|\left\langle f_{n} \mid u(0)\right\rangle\right|^{2}, \quad t \ll \frac{1}{\lambda_{1}}, \frac{1}{2 \ell_{\max } \omega_{o}} .
\end{aligned}
$$

Here $u(0)$ is the total velocity field $u(t)$ evaluated at time $t=0$. This expression becomes problematic to apply in the case where infinitely many harmonics of the velocity field is present at $t=0$ as for the startup of the Poiseuille flow, but for flows with a maximum frequency we have shown $D_{\text {eff }}(t)>0$.

\section{A.4. Numerics}

For numerical evaluations of the effective diffusivity $D_{\text {eff }}(t)$ we relied on three methods. First, using the commercial finite element package COMSOL MULTIPHYSICS version 3.5a, we calculated $D_{\text {eff }}(t)$ numerically directly from the definition (2.11) by solving for the concentration field $c$ in the governing advection-diffusion equation (2.9) using the analytical solutions for the velocity field as input. From the obtained $c(\boldsymbol{r}, t)$ we determined $M_{1}(t), \mathrm{d} M_{1}(t) / \mathrm{d} t$ and $\mathrm{d} M_{2}(t) / \mathrm{d} t$, and from these $D_{\text {eff }}(t)$. To ensure mass conservation the number of mesh elements was chosen so that the local Péclet number in each cell (as given by the velocity in the mesh element, the mesh element length and the global diffusivity) was approximately 0.5 . Where possible, the available symmetries were exploited for computational efficiency. To this end the solutions of the flow problems in channels of circular cross-section took advantage of the azimuthal symmetry and the solution was computed in a frame of reference travelling with the mean speed of the flow.

Second, for rectangular cross-sections the problem was only solved for one quarter of the cross-section in a frame of reference moving with the flow mean speed. However, due to memory limitations even these simplifications proved unfeasible for Péclet numbers exceeding $\sim 2$ and aspect ratios above $\sim 2$. Thus, for the results presented in figure $3(a)$ we numerically solved the axial moment equation $(2.12 b)$ 
for $c_{o}$ and $c_{1}$, and from these obtained $M_{1}(t), \mathrm{d} M_{1}(t) / \mathrm{d} t$, and $\mathrm{d} M_{2}(t) / \mathrm{d} t$, as well as $D_{\text {eff }}(t)$. Time averages of the numerical simulation results were computed by running the simulation for a full period of the base frequency after the decay of all transients, followed by numerical integration of $D_{\text {eff }}(t)$ over the period.

Third, we calculated $D_{\text {eff }}(t)$ based on our theoretical expression (3.22), but reduced the number of terms needed to be calculated by separating the $\ell$ - and $k$-sums and collecting complex conjugated pairs of index $\{\ell,-\ell\}$ and $\{k,-k\}$,

$$
\begin{aligned}
D_{\text {eff }}(t)= & 1+P e^{2} \sum_{n=1}^{\infty}\left\{\frac{\left\langle f_{n} \mid u_{0}\right\rangle}{\lambda_{n}}\left[1-\mathrm{e}^{-\lambda_{n} t}\right]+2 \sum_{\ell=1}^{\infty} \operatorname{Re}\left[\frac{\left\langle f_{n} \mid u_{\ell}\right\rangle}{\lambda_{n}+\mathrm{i} \ell \omega_{o}}\left(\mathrm{e}^{\mathrm{i} \ell \omega_{o} t}-\mathrm{e}^{-\lambda_{n} t}\right)\right]\right\} \\
& \times\left\{\left\langle f_{n} \mid u_{0}\right\rangle+2 \sum_{k=1}^{\infty} \operatorname{Re}\left[\left\langle f_{n} \mid u_{k}\right\rangle \mathrm{e}^{\mathrm{i} k \omega_{o} t}\right]\right\} .
\end{aligned}
$$

Furthermore, the involved $n$-, $\ell$ - and $k$-dependent terms are calculated and placed in lists before evaluating the sums. The latter converge quickly, and we have therefore truncated them after the first 50 terms unless stated otherwise in the text.

\section{REFERENCES}

Ajdari, A., Bontoux, N. \& Stone, H. A. 2006 Hydrodynamic dispersion in shallow microchannels: the effect of cross-sectional shape. Analyt. Chem. 78, 387-392.

ARIS, R. 1956 On the dispersion of a solute in a fluid flowing through a tube. Proc. R. Soc. Lond. A Mat. 235 (1200), 67-77.

ARIS, R. 1960 On the dispersion of solute in pulsating flow through a tube. Proc. R. Soc. Lond. A Mat. 259 (1298), 370-376.

BANDYOPADHYAY, S. \& MAZUMder, B. S. 1999 Unsteady convective diffusion in a pulsatile flow through a channel. Acta Mechanica 134, 1-16.

BArton, N. G. 1983 On the method of moments for solute dispersion. J. Fluid Mech. 126, 205-218.

Batchelor, G. K. 1967 An Introduction to Fluid Dynamics. Cambridge University Press.

Bird, R. B., Stewart, W. E. \& Lightfoot, E. N. 2006 Transport Phenomena, 2nd edn. John Wiley and Sons.

Bontoux, N., Pépin, A., Chen, Y., Ajdari, A. \& Stone, H. A. 2006 Experimental characterization of hydrodynamic dispersion in shallow microchannels. Lab on a Chip $\mathbf{6}$, 930-935.

Brenner, H. \& Edwards, D. A. 1993 Macrotransport Processes. Butterworth-Heinemann.

BRUUS, H. 2008 Theoretical Microfluidics. Oxford University Press.

BruUs, H. \& Stone, A. D. 1994 Quantum chaos in a deformable billiard: applications to quantum dots. Phys. Rev. B 50 (24), 18275-18287.

Camassa, R., Lin, Z. \& McLaughlin, R. 2010 The exact evolution of scalar variance in pipe and channel flow. Commun. Math. Sci. 8 (2), 601-626.

Chatwin, P. C. 1975 On the longitudinal dispersion of passive contaminant in oscillatory flows in tubes. J. Fluid Mech. 71, 513-527.

Chatwin, P. C. 1977 Initial development of longitudinal dispersion in straight tubes. J. Fluid Mech. 80, 33-48.

Chatwin, P. C. \& Sullivan, P. J. 1982 The effects of aspect ratio on longitudinal diffusivity in rectangular channels. J. Fluid Mech. 120, 347-358.

DiRAC, P. A. M. 1981 The Principles of Quantum Mechanics, 4th edn. Oxford University Press.

Doshi, M. R., DAIYA, P. M. \& GILL, W. N. 1978 Three dimensional laminar dispersion in open and closed rectangular conduits. Chem. Engng Sci. 33, 795-804.

Dutta, D., Ramachandran, A \& Leighton, D. T. 2006 Effect of channel geometry on solute dispersion in pressure-driven microfluidic systems. Microfluid Nanofluid 2, 275-290. 
Erdogan, M. E. \& Chatwin, P. C. 1967 Effects of curvature and buoyancy on laminar dispersion of solute in a horizontal tube. J. Fluid Mech. 29 (3), 465-484.

FAN, L. T. \& WANG, C. B. 1966 Dispersion of matter in non-Newtonian laminar flow through a circular tube. Proc. R. Soc. Lond. A Mat. 292 (1429), 203-208.

GleEson, J. P. 2002 Electroosmotic flows with random zeta potential. J. Colloid Interface Sci. 249 (1), 217-226.

GODDARD, J. D. 1993 The Green's function for passive scalar diffusion in a homogeneously sheared continuum. Phys. Fluids A 5, 2295-2297.

HARris, H. G. \& Goren, S. L. 1967 Axial diffusion in a cylinder with pulsed flow. Chem. Engng Sci. 22, 1571-1576.

Jansons, K. M. 2006 On Taylor dispersion in oscillatory channel flows. Proc. R. Soc. Lond. A Mat. 462, 3501-3509.

LAtini, M \& Bernoff, AJ 2001 Transient anomalous diffusion in Poiseuille flow. J. Fluid Mech. 441, 399-411.

LEIGHTON, D. T. 1989 Diffusion from an intial point distribution in an unbounded oscillating simple shear flow. Physico-Chem. Hydrodyn. 11, 377-386.

LIDE (EDITOR-IN-CHIEF), D. R. 1995 CRC Handbook of Chemistry and Physics, 75th edn. CRC Press.

Menta, M. L. 2004 Random Matrices, 3rd edn. Pure and Applied Mathematics, vol. 142. Elsevier/Academic Press.

Molloy, R. F. \& Leighton, D. T. 1998 Binary oscillatory cross-flow electrophoresis: theory and experiments. J. Pharma. Sci. 87, 1270-1281.

Mortensen, N. A. \& BruUs, H. 2006 Universal dynamics in the onset of a Hagen-Poiseuille flow. Phys. Rev. E 74 (1), 017301.

Mortensen, N. A., Olesen, L. H. \& BRUus, H. 2006 Transport coefficients for electrolytes in arbitrarily shaped nano and micro-fluidic channels. New J. Phys. 8, 37-51.

Mukherjee, A. \& Mazumder, B. S. 1988 Dispersion of contaminant in oscillatory flows. Acta Mechanica 74, 107.

Paul, S. \& Mazumder, B. S. 2008 Dispersion in unsteady Couette-Poiseuille flows. Intl J. Engng Sci. 46, 1203-1217.

Probstein, R. F. 1994 Physicochemical Hydrodynamics. An Introduction, 2nd edn. John Wiley and Sons.

SANKARASUbRAmANiAn, R. \& GiLl, W. N. 1973 Unsteady convective diffusion with interphase mass-transfer. Proc. R. Soc. Lond. A Mat. 333 (1592), 115-132.

Skafte-Pedersen, P., Sabourin, D., Dufva, M. \& Snakenborg, D. 2009 Multi-channel peristaltic pump for microfluidic applications featuring monolithic PDMS inlay. Lab on a Chip 9, 3003-3006.

TAYLOR, G. I. 1953 Dispersion of soluble matter in solvent flowing slowly through a tube. Proc. R. Soc. Lond. A Mat. 219 (1137), 186.

TAYLOR, H. M. \& LEONARD, E. F. 1965 Axial dispersion during pulsating pipe flow. AIChE J. 11 (4), 686-689.

Thomas, A. M. \& Narayanan, R. 2001 Physics of oscillatory flow and its effect on the mass transfer and separation of species. Phys. Fluids 13 (4), 859-866.

VAN DEN BROECK, C. 1982 A stochastic description of longitudinal dispersion in uniaxial flows. Physica A 112, 343-352.

Vedel, S., Olesen, L. H. \& BRuus, H. 2010 Pulsatile microfluidics as an analytical tool for determining the dynamic characteristics of microfluidic systems. J. Micromech. Microengng 20, 035026.

Vikhansky, A. \& WANG, W. 2011 Taylor dispersion in finite-length capillaries. Chem. Engng Sci. 66 (4), 642-649.

WATSOn, E. J. 1983 Diffusion in oscillatory pipe flow. J. Fluid Mech. 133, 233-244.

Womersley, J. R. 1955 Method for the calculation of velocity, rate of flow and viscous drag in arteries when the pressure gradient is known. J. Physiol. 127, 553-563. 